\title{
Symptome, Komorbiditäten und Therapie von Kindern und Jugendlichen mit Geschlechtsdysphorie
}

\author{
Dissertation \\ zur Erlangung des akademischen Grades \\ Dr. med.
}

an der Medizinischen Fakultät

der Universität Leipzig

eingereicht von: Annika Alica Specht

Geburtsdatum / Geburtsort: 09.10.1992 in Mönchengladbach

angefertigt an / in: Universität Leipzig, Medizinische Fakultät

Betreuer: Prof. Dr. med. Wieland Kiess

Beschluss über die Verleihung des Doktorgrads vom: 21.09.2021 
Inhaltsverzeichnis.

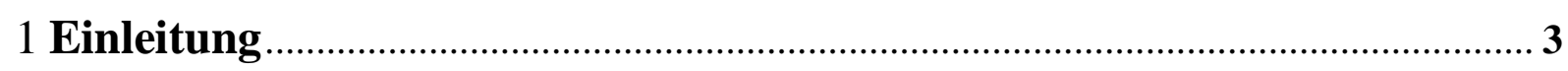

1.1 Geschlechtsdysphorie im Kindes- und Jugendalter ............................................... 3

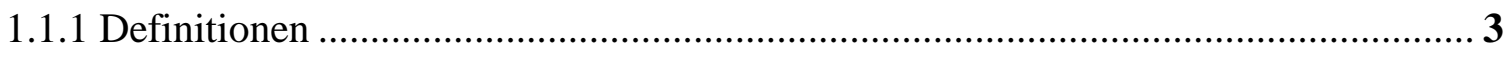

1.1.2 Prävalenz und Geschlechterverhältnis .......................................................... 4

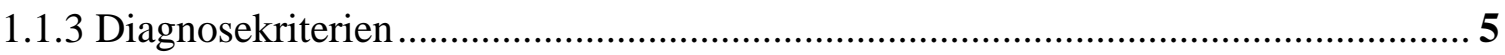

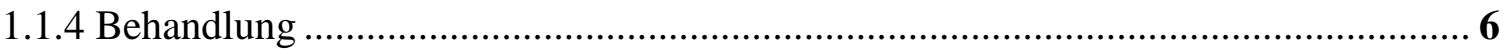

1.1.4.1 Mögliche Nebenwirkungen der pubertätshemmenden Therapie mit GnRHAnaloga

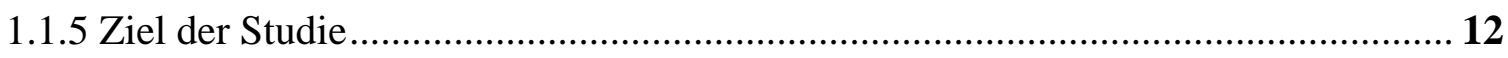

2 Publikationsmanuskript .............................................................................. 13

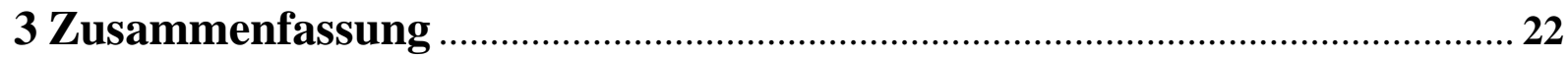

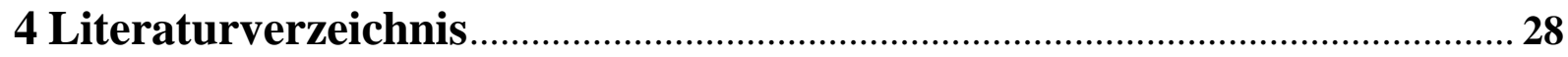

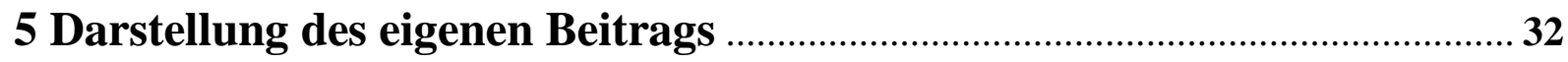

6 Erklärung über die eigenständige Abfassung der Arbeit .............................. 34

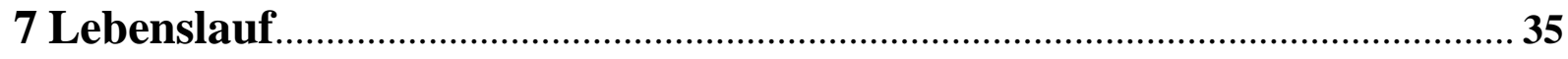

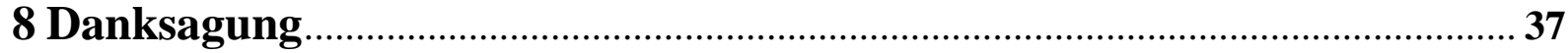




\section{Einleitung}

\subsection{Geschlechtsdysphorie im Kindes- und Jugendalter}

\subsubsection{Definition}

Geschlechtsdysphorie im Kindes- und Jugendalter (GD) ist sowohl in „Diagnostic and Statistical Manual of Mental Disorders - Fifth Editon (DSM - V) “ [2] als auch in , The ICD-10 Classification of Mental and Behavioural Disorders " [34] gelistet und wird wie folgt definiert:

Störung der Geschlechtsidentität im Kindesalter (F64.2):

Diese Störung geht mit einem ausgeprägten Unbehagen oder gar mit einem Verleugnen des eigenen Geschlechts seitens des Kindes einher. In der Regel wird die Diagnose in früher Kindheit gestellt, in jedem Fall aber vor Beginn der Pubertät.

Von diesen im ICD-10 und DSM-V kodierten Erkrankungen sind noch weitere in der Fachliteratur verwendete Begriffe abzugrenzen und $\mathrm{zu}$ definieren. Hierbei ist es schwierig, eng verwandte und teils auch synonym verwendete Begriffe voneinander abzugrenzen, da diese sich sowohl in der Fachliteratur als auch in der Szene in ständigem Wandel befinden. Daher haben die nachfolgenden Begriffsdefinitionen keinen allgemeingültigen Charakter, sondern definieren lediglich die in dieser Promotion verwendeten Termini.

- Geschlechtsdysphorie (GD): Ein wachsendes Unbehagen in Bezug auf das biologische Geschlecht, früher als Geschlechtsidentitätsstörung (GID) bezeichnet, aber sowohl in der neuen S3-Leitlinie als auch dem WPATH zugunsten der GD abgeändert. - Geschlechtsinkongruenz: Diskrepanz zwischen der Geschlechtsidentität bzw. dem gelebten/erlebten Geschlecht und den körperlichen Geschlechtsmerkmalen. 
- Biologisches Geschlecht oder Geburtsgeschlecht: Das biologische Geschlecht bezeichnet das bei Geburt festgestellte Geschlecht (i.d.R. das chromosomale Geschlecht).

Gelebtes/erlebtes Geschlecht: Das präferierte und/oder erlebte und gelebte Geschlecht, welches ein anderes oder alternatives Geschlecht zum biologischen Geschlecht darstellt.

\subsubsection{Prävalenz und Geschlechterverhältnis}

Es existiert bisher noch keine Studie aus Deutschland, die die Prävalenz der Geschlechtsdysphorie (GD) im Kindes- und Jugendalter ermitteln konnte. Die Studie von Bosinski et al. [6] gibt allerdings einen Anhalt. In der Studie wurden Fragebögen an Kinderärzt*innen, Kinder- und Jugendpsychiater*innen, Kinderpsycholog*innen und Beratungsstellen in Schleswig-Holstein verschickt, durch welche ermittelt wurde, wie viele Kinder und Jugendliche mit GD in den letzten fünf Jahren vorstellig waren. Zusammengefasst wurde bei 326 Kindern eine GD diagnostiziert. In SchleswigHolstein lag der Anteil der unter 14-Jährigen an der Gesamtbevölkerung bei 14,5\%, also 377.000. Somit könnte man auf die Prävalenz schließen, allerdings ist dieses Ergebnis nur als ungefährer Wert zu betrachten, da in der Studie darauf hingewiesen wird, dass Doppelnennungen wahrscheinlich seien und man nicht sicher gehen könne, dass alle an GD leidenden Kinder im betrachteten Zeitraum vorstellig geworden seien. Eine neuere Studie aus den Niederlanden beschäftigt sich ebenso mit der Prävalenz der Geschlechtsdysphorie [22]. Es wurde herausgefunden, dass 4,6\% der biologisch männlichen und 3,2\% der biologisch weiblichen Befragten sich genauso mit dem anderen Geschlecht identifizieren können wie mit ihren jetzigen. 1,1\% der biologischen Männer und $0,8 \%$ der biologischen Frauen gaben an, sich mehr mit dem anderen Geschlecht $\mathrm{zu}$ identifizieren, wobei nur $0,6 \%$ bzw. 0,2\% der Befragten eine Hormontherapie oder Operation in Betracht ziehen.

Bosinski et al. [6] ermittelte außerdem das Verhältnis von Jungen mit GD zu Mädchen mit GD ausgehend vom Geburtsgeschlecht. Im präpubertären Alter ergab sich ein Verhältnis von 16:6, im Pubertätsalter stellte sich nahezu ein Verhältnis von 1:1 ein. Die Theorie, dass sich das Verhältnis zwischen den Geschlechtern im Laufe der Pubertät angleicht, konnte durch mehrere Studien belegt werden [6, 8, 28, 33]. Studien aus Behandlungszentren in Toronto [33] und Amsterdam [8] zeigen, das sich das 
Geschlechterverhältnis auch über die Zeit verändert hat. Während in der Zeit vor 2000 das Geschlechterverhältnis auf das Geburtsgeschlecht bezogen von Jungen zu Mädchen mit GD in Toronto bei 5,75:1 und in Amsterdam bei 2,93:1 lag, näherte es sich nach 2000 in Toronto auf 3,41:1 und in Amsterdam auf 1,68:1 (2008 - 2011) [28] an. Diese Theorie wird durch eine deutsche Studie von Garrels et al. [15] und anderen europäischen Studien [12, 23, 30] bestärkt. Neuste Untersuchungen sprechen gar von einer Umkehr des Geschlechterverhältnisses [7, 29].

1.1.3 Diagnosekriterien der Geschlechtsdysphorie bei Kindern und Jugendlichen nach DSMV-Kriterien [2]

In der Tabelle sind die Diagnosekriterien nach dem DSM-V für Kinder und Jugendliche bzw. Erwachsene zusammengefasst:

Kinder
A. Eine deutliche Unstimmigkeit zwischen
dem gelebten/erlebten Geschlecht und dem
biologischen Geschlecht über einen Zeitraum
von 6 Monaten hinweg, welche sich durch
mindestens 6 der folgenden Kriterien
manifestiert (eines muss Kriterium A1 sein):

1. Starkes Unbehagen bezüglich des biologischen Geschlechts oder das Bestehen darauf, dem anderen Geschlecht (oder einem alternativen Geschlecht, das nicht dem biologischen Geschlecht entspricht) anzugehören.

2. Bei biologischen Jungen: deutliches Bevorzugen gegengeschlechtlicher und femininer Kleidung; bei biologischen Mädchen: deutliches Bevorzugen maskuliner Kleidung und ein striktes Ablehnen typisch femininer Kleidung.

3. Deutliches Präferieren gegengeschlechtlicher Rollen in Fantasiespielen und Rollenspielen.

4. Deutliches Bevorzugen von Spielzeug, Spielen und Hobbies, welche typischerweise dem anderen Geschlecht zugeordnet werden.

\section{Jugendliche und Erwachsene}

A. Eine deutliche Unstimmigkeit zwischen dem gelebten/erlebten Geschlecht und dem biologischen Geschlecht über einen Zeitraum von 6 Monaten hinweg, welche sich durch mindestens 2 der folgenden Kriterien manifestiert:

1. Starkes Unbehagen bezüglich des gelebten/erlebten Geschlechts und dem primären und /oder sekundären Geschlechtsmerkmalen (oder bei jungen Jugendlichen bezüglich der erwarteten sekundären Geschlechtsmerkmale).

2. Starker Wunsch, die primären und /oder sekundären

Geschlechtsmerkmale zu verlieren, da diese die Unstimmigkeit zu dem gelebten/erlebten Geschlecht (oder einem alternativen Geschlecht, das nicht dem biologischen Geschlecht entspricht) hervorheben.

3. Starker Wunsch, primäre und /oder sekundäre Geschlechtsmerkmale des anderen Geschlechts zu haben.

4. Starker Wunsch vom anderen Geschlecht (oder einem alternativen Geschlecht, das nicht dem biologischen Geschlecht entspricht) zu sein. 
5. Bevorzugen gegengeschlechtlicher Freunde.

6. Bei biologischen Jungen: eine starke Ablehnung typisch maskuliner Spiele, Spielzeuge und Hobbies und das Vermeiden von Raufen und Toben; bei biologischen Mädchen: eine starke Ablehnung typisch femininer Spiele, Spielzeuge und Hobbies.

7. Starke Ablehnung des eigenen biologischen Geschlechts.

8. Starker Wunsch, dass die primären und /oder sekundären Geschlechtsmerkmale zu dem gelebten/erlebten Geschlechts passen.

B. Das Kriterium muss mit einem signifikanten Stress und einer Beeinträchtigung der Soziallebens, der Schule oder anderen wichtigen Lebensbereichen einher gehen.
5. Starker Wunsch, wie das andere Geschlecht (oder ein alternatives Geschlecht, das nicht dem biologischen Geschlecht entspricht) behandelt zu werden.

6. Überzeugung, dass man typische Gefühle hat und Verhaltensmuster lebt wie das andere Geschlecht (oder ein alternatives Geschlecht, das nicht dem biologischen Geschlecht entspricht).

B. Das Kriterium muss mit einem signifikanten Stress und einer Beeinträchtigung des Soziallebens, des Berufs oder anderen wichtigen Lebensbereichen einher gehen.

Geschlechtsdysphorie nach DSM-V [2]

\subsubsection{Behandlung}

Die „World Professional Association of Transgender Health's Standards of Care (WPATH-SOC)“ [10] (früher auch „The Harry Benjamin International Gender Dysphoria Association's Standards of Care for Gender Identity Disorders“"genannt) ist eine internationale Richtlinie für die Behandlung von transsexuellen Erwachsenen und Kindern mit Geschlechtsdysphorie. Sie besteht aus fünf Säulen: der Alltagstestphase, der Therapie mit GnRH-Analoga, der Therapie mit geschlechtsangleichenden Hormonen, der operativen Therapie und der begleitenden Psychotherapie.

Ziele der Psychotherapie

Zunächst ist es notwendig, dass durch Psycholog*innen eine gute und lückenlose Diagnostik durchgeführt wird. Wichtig ist dies besonders unter der Berücksichtigung von Studien, die die Patientenzufriedenheit nach Therapiebeginn ermittelten [24]. Es zeigte sich, dass eine Therapieunzufriedenheit oder ein Therapieabbruch bisher nur von 
Patient*innen berichtet wurde, bei welchen ohnehin Zweifel an der Richtigkeit der Diagnose bestanden haben. Daher ist es notwendig, andere psychologische oder somatische Erkrankungen auszuschließen, bevor die Diagnose einer Geschlechtsdysphorie im Kindes- und Jugendalter gestellt wird.

Bei Kindern und Jugendlichen muss außerdem auf psychiatrische Komorbiditäten geachtet werden, da durch mehrere Studien belegt werden konnte, dass besonders Kinder und Jugendliche sexueller Minderheiten (LGBT: Lesbian, gay, bisexual and transgender) zu Depressionen, selbstverletzendem Verhalten oder Suizidversuchen [14, 16, 25] neigen. Die Psychotherapie sollte sich daher besonders auf die Behandlung dieser Komorbiditäten konzentrieren. Durch Informationen über Beratungsangebote (z.B. Selbsthilfegruppen) sollte versucht werden, den emotionalen Stress, den die GD bei vielen Betroffenen auslöst, zu lindern.

Der behandelnden Fachkräfte sollte auch Hilfestellung im Alltag anbieten. Wichtig ist zusätzlich zur Psychotherapie des Kindes bzw. des Jugendlichen das Angebot einer Familientherapie, da häufig Ängste und Unsicherheiten bei den Eltern im Umgang mit ihrem Kind existieren, die aufgearbeitet werden müssen, um stabiles und unterstützendes Umfeld zu schaffen. Eine Studie von Grossman und D'Augelli zeigte, dass $35 \%$ bis $73 \%$ der Kinder und Jugendlichen gelegentlich bis häufig verbalen Missbrauch in Form von Beschimpfungen, Kritik, Bloßstellung vor anderen und Beschuldigungen aufgrund ihrer Sexualität durch ihre Eltern erfahren haben. $13 \%$ bis $36 \%$ der Befragten berichten sogar von regelmäßigem körperlichen Missbrauch. Kinder und Jugendliche, die von solcher Gewalt durch die Eltern berichtet haben, begingen häufiger Suizidversuche [16].

Darüber hinaus benötigen viele Jugendliche bzw. deren Familien Unterstützung im Umgang mit dem sozialen Umfeld. Besonders die Frage, in welchem Alter das „Coming-out" stattfinden sollte, ist schwierig zu beantworten. Da die Persistenzrate der Geschlechtsdysphorie relativ niedrig ist $[13,31]$ und Erfahrungen zeigen, dass die „Wiedereingliederung“ eines geschlechtsnichtkonformen Kindes in die Geschlechterrolle seines biologischen Geschlechts durch sozialen Druck erschwert oder sogar verzögert werden kann [27], muss genau abgewogen und überdacht werden, wann der richtige Zeitpunkt ist und in welchem Umfang das soziale Umfeld informiert werden soll. Viele Jugendliche berichten außerdem von Mobbing oder gar körperlichen Angriffen aufgrund ihrer Geschlechtsdysphorie, weshalb auch hier psychologische Betreuung notwendig sein kann [17]. 
Die Psychotherapie bei Kindern und Jugendlichen mit Geschlechtsdysphorie endet nicht mit der Diagnosestellung „Geschlechtsdysphorie“, sondern sollte auf jeden Fall über den Zeitraum einer eventuellen medikamentösen oder chirurgischen Therapie hinaus andauern.

Therapiekonzept der Geschlechtsdysphorie

Die Therapie der GD gliedert sich bei Kindern und Jugendlichen in vier Abschnitte.

Alltagstestphase

Zunächst beginnt man mit der Alltagstestphase, in der die gegengeschlechtliche Rolle im alltäglichen Leben übernommen wird, welche oft zeitgleich oder überschneidend zur reversiblen Therapie stattfindet. Wenn sich die GD schon sehr früh gezeigt hat, leben viele Jugendliche bereits in der gegengeschlechtlichen Rolle, weshalb nach der obligaten sechsmonatigen psychologischen oder psychiatrischen Betreuung direkt mit der reversiblen Therapie begonnen werden kann.

Reversible Therapie

Voraussetzungen für die Therapie sind eine diagnostizierte GD, welche anhaltend und mit dem Einsetzen der Pubertät zunehmend ist sowie eine vorangegangene Erfassung der psychischen Gesundheit, die die Eignung und psychische Stabilität des Jugendlichen zur Therapie bestätigt. Da die Patient*innen i.d.R. bei Beginn der Therapie noch unter 16 Jahre alt sind, bedarf es ebenso einer Zustimmung und Einbeziehung der Eltern bzw. des Erziehungsberechtigten in die Behandlung.

Es gibt keine klare Altersgrenze, ab wann die hormonelle Therapie bei Jugendlichen erlaubt bzw. indiziert ist. Auch die WPATH-SOC gibt hierzu keine genauen Angaben. Richtlinien aus den Niederlanden, die sich auch an der WPATH-SOC orientieren, haben aufgrund des besseren Verständnisses der Pubertät und Studien, die belegen, das sowohl das psychische als auch das operative Outcome der Geschlechtsumwandlung besser ist, wenn die Therapie früh begonnen wird, ein Mindestalter von 12 Jahren bzw. Pubertätsstadium 2 bis 3 nach Tanner festgelegt [11]. Der WPATH-SOC ist dahingegen nicht eindeutig. Dort wird lediglich empfohlen, das Pubertätsstadium Tanner 2 
abzuwarten, wobei eingeräumt wird, dass es auch Kinder gibt, die jenes im Alter von beispielsweise 9 Jahren erreichen.

Eine Möglichkeit der Therapie sind GnRH-Analoga (Gonadotropin-releasing hormoneAnaloga), welche die Testosteronsekretion bzw. Östrogen- und Progesteronbildung unterbinden. Für beide Geschlechter stellt die Behandlung mit GnRH-Analoga Therapie der ersten Wahl dar [18]. Die Behandlung mit GnRH-Analoga bewirkt ein Sistieren der Pubertätsentwicklung oder sogar eine teilweise oder komplette Rückbildung der bereits vorhandenen sekundären Geschlechtsmerkmale. Therapieziel ist zum einen die Reduktion des Leidesdrucks, welcher bei Jugendlichen mit GD durch die Ausbildung der sekundären Geschlechtsmerkmale im Zuge der Pubertät deutlich zunehmen kann [27] und die Möglichkeit, mehr Zeit zur Verfügung zu haben, um den Wunsch der Geschlechtsänderung $\mathrm{zu}$ überdenken. Zum anderen ermöglicht der frühe Therapiebeginn mit GnRH-Analoga ein besseres chirurgisches und psychologisches Ergebnis nach Beendigung der Therapie [11].

Teilweise reversible Therapie

Dieser Therapieschritt beinhaltet die Behandlung mit geschlechtsangleichenden Hormonen. Voraussetzung für den Therapiestart ist in vielen europäischen Ländern ein Mindestalter von 16 Jahren, das als Beginn der medizinischen Mündigkeit gesehen [10, 11]. Auch in Deutschland gilt 16 als empfohlenes Alter [24]. Die Zustimmung der Eltern ist somit nicht zwingend notwendig, aber äußerst empfehlenswert, um die Unterstützung der Jugendlichen durch ihr soziales Umfeld zu sichern. Im WPATH-SOC ist keine eindeutige Altersgrenze oder ein genauer Therapieplan benannt. Es wird lediglich darauf hingewiesen, dass der Behandlungsplan sich deutlich von dem für Erwachsene unterscheidet und die Hormontherapie für Jugendliche so angepasst werden soll, „dass sie der somatischen, emotionalen und mentalen Entwicklung, die während des Erwachsenwerdens stattfindet, gerecht wird“" [10]. Bisher fehlt eine S3-Leitlinie für die Therapie von Kindern und Jugendlichen mit Geschlechtsdysphorie. Im Oktober 2018 erschien aber eine S3-Leitlinie für Erwachsene mit Geschlechtsdysphorie [3]. Eine S3Leitlinie für die Diagnostik und Behandlung der Geschlechtsdysphorie im Kindes- und Jugendalter ist angemeldet [4].

Irreversible, chirurgische Therapie 
Die Voraussetzung für die chirurgische Geschlechtsangleichung ist die Vollendung des 18. Lebensjahres. Außerdem sollte der Jugendliche schon mindestens 12 Monate in der gegengeschlechtlichen Rolle gelebt haben. Die WPATH-SOC betonen dabei ausdrücklich, dass das Überschreiten der Altersgrenze von 18 Jahren nicht als eine Indikation zur chirurgischen Behandlung der GD gesehen werden darf, sondern lediglich als Voraussetzung [10]. Bei Female-to-Male-Patienten beinhaltet die operative Geschlechtsangleichung als ersten Schritt die Mastektomie mit anschließendem Aufbau einer männlichen Brust. Eine Mastektomie kann schon vor dem 18. Lebensjahr stattfinden, sollte das Erleben der männlichen Geschlechtsrolle ohne nicht möglich sein. Patienten sollten bereits eine angemessene Zeit in der gewünschten Geschlechtsrolle gelebt und ein Jahr eine Testosteronbehandlung bekommen haben. Daran schließt sich die Genitalplastik an. Diese besteht zunächst aus der Entfernung der Gebärmutter und der Adnexen. Die Konstruktion der männlichen Genitalien besteht aus einer Rekonstruktion der Harnröhre, gegebenenfalls in Kombination mit einer Metaidoioplastik oder Phalloplastik, einer Skrotoplastik sowie der Implantation einer Hoden- und/oder Penisprothese. Bei Male-to-Female-Patientinnen sieht die operative Geschlechtsangleichung nach der Entfernung von Penis und Hoden eine Vaginalplastik, Klitorisplastik und eine Vulvaplastik vor. Sollte das Brustwachstum durch die Behandlung mit Östrogenen nicht zufriedenstellend sein, kann auch ein chirurgischer Brustaufbau durchgeführt werden.

Während das Ergebnis der Genitalplastik bei Male-to-Female-Patientinnen häufig zufriedenstellend ist, ist das Ergebnis bei Female-to-Male-Patienten besonders bezüglich der Fähigkeit zum Geschlechtsverkehr häufig noch nicht zufriedenstellend. Die hypertrophe Klitoris ist oft $\mathrm{zu}$ klein, um einen ausreichend großen Penis zu konstruieren, weshalb einige Female-to-Male-Patienten mit der chirurgischen Geschlechtsangleichung warten möchten, bis sich bessere Operationsverfahren etabliert haben [11]. Obwohl sowohl die Metaidoioplastik als auch die Phalloplastik mit schwerwiegenden Komplikationen verbunden sein kann (z.B. Harnwegsstenosen oder Vernarbungen), zeigen Studien, dass die Zufriedenheit der Patienten, die sich einer operativen Geschlechtsangleichung unterziehen, höher ist als die Zufriedenheit derer, die noch auf die Operation warten [21].

1.1.4.1 Mögliche Nebenwirkungen der pubertätshemmenden Therapie mit GnRHAnaloga 
Nicht zuletzt wegen befürchteter Nebenwirkungen wird die Therapie mit GnRHAnaloga zur Hemmung der Pubertät bei Jugendlichen mit einer Geschlechtsdysphorie kontrovers diskutiert. Eine Studie von Delemarre-van der Waal et al. [11] beschäftigte sich mit den Auswirkungen der GnRH-Analoga-Therapie auf den Körper. Dabei untersuchte man die Wirksamkeit der Therapie mit GnRH-Analoga und den gegengeschlechtlichen Hormonen, die Entwicklung der Knochendichte und das damit verbundene Risiko für Osteoporose, metabolische Parameter (Nüchternglukosewert, Insulinspiegel, HDL, LDL, Cholesterin) und die Entwicklung der Körperkonstitution. Die Teilnehmer der Studie waren mindestens zwei Jahre in Behandlung. Es ließen sich keine signifikanten negativen Auswirkungen der GnRH-Analoga- oder der geschlechtsangleichenden Hormontherapie ausmachen. Des Weiteren berichteten die Befragten eine große Zufriedenheit mit der hormonellen Therapie, weshalb die Autoren darauf schlossen, dass bei Kindern mit einer früh diagnostizierten GD eine Behandlung mit GnRH-Analoga zu rechtfertigen sei. Durch die Verzögerung der Pubertät werde den betroffenen Kindern, Eltern und betreuende Psycholog*innen bzw. Ärzt*innen mehr Zeit gegeben, um die weitreichende Entscheidung über eine Geschlechtsumwandlung abzuwägen. Diese Zeit fehle, wenn die Kinder in die Pubertät kämen. Der Fokus verschiebe sich durch den zunehmenden Leidensdruck, der mit der Ausbildung sekundärer Geschlechtsmerkmale einher gehe, auf die möglichst schnelle Problemlösung, nämlich die Änderung des Geschlechts.

Korte et al. [20] stellen in ihrer Arbeit die Hypothese auf, dass die Therapie mit GnRHAnaloga die Geschlechtsidentitätsfindung beeinflussen könne. Die Annahme, dass die Geschlechtsidentitätsfindung mit 12 noch nicht abgeschlossen sei, wird mit der niedrigen Anzahl an Jugendlichen, bei denen die GD über das Kindes- bzw. Jugendalter hinaus fortbesteht, begründet. Nur zwei bis $25 \%$ der Kinder und Jugendlichen, bei denen eine GD diagnostiziert wurde, zeigen nach Korte et al. eine anhaltende transsexuelle Entwicklung. Außerdem seien keine eindeutigen diagnostischen Marker vorhanden, die die Wahrscheinlichkeit eines Fortbestehens der GD valide einschätzen können.

Meyenburg führt in seiner Studie allerdings diagnostische Kriterien an, die es den behandelnden Psycholog*innen bzw. Psychiater*innen ermöglichen sollen, die Geschlechtsdysphorie von anderen sexuellen Störungen oder passageren Geschlechtsdysphorien zu unterscheiden. Diese Kriterien lauten wie folgt: „(1) Eindeutig erfüllte diagnostische Hauptkriterien des unbedingten Wunsches, dem 
Gegengeschlecht vollständig anzugehören und die unbedingte Ablehnung des eigenen Körpergeschlechts, (2) durchgehendes Bestehen der Transidentität seit frühester Kindheit sowie (3) der überzeugende Eindruck beim Erstkontakt, einer Person des Gegengeschlechts gegenüberzusitzen" [24]. Auch diese Studie konnte eine hohe Zufriedenheit der Patienten und deren Eltern in Bezug auf die frühe hormonelle Therapie evaluieren. Unzufriedenheit seitens der Patient*innen wurde lediglich durch solche berichtet, bei denen die Spezialist*innen ohnehin Zweifel an der Richtigkeit der Diagnose hatten.

Die Studie von Steensma et al. [27] befragte 53 Jugendliche, bei denen eine Geschlechtsdysphorie diagnostiziert wurde, um die Entwicklungsunterschiede zwischen Jugendlichen mit persistierender und desistierender GD sowie deren psychosoziale Entwicklung zu untersuchen. 24 der 53 befragten Jugendlichen waren nach der Erstdiagnose nicht wieder bei Ärzt*innen vorstellig, bei den Übrigen 29 bestand die GD weiterhin, manche befanden sich bereits in Behandlung. In den geführten Interviews wurde deutlich, dass die Altersspanne, in der sich entscheidet, ob die GD besteht oder nicht, zwischen 11 bis 13 Jahren liegt. Somit widerspricht dieses Ergebnis der Annahme von Korte et al., dass die Geschlechtsidentität erst im Zuge oder mit Abschluss der Pubertät erreicht werden könne.

\subsubsection{Ziel der Studie}

Ziel der Studie ist es, einen Überblick über die Betreuung und Therapie, aber auch das soziale und familiäre Umfeld dieser noch sehr wenig betrachteten Patientengruppe zu geben. So soll ein Beitrag dazu geleistet werden, die Einschätzung und die klinische Versorgung von Kindern und Jugendlichen mit Geschlechtsdysphorie zu verbessern. Dabei soll im Speziellen der Fokus auf die Notwenigkeit der interdisziplinären Betreuung der Kinder und Jugendlichen gelegt werden. 


\title{
2 Publikationsmanuskript
}

Online publiziert: 17.12 .2019

Origlnalarbelt Thieme

\section{Symptome, Komorbiditäten und Therapie von Kindern und Jugendlichen mit Geschlechtsdysphorie}

\author{
Symptoms, Comorbidities and Therapy of Children and
} Adolescents with Gender Dysphoria

Autoren

Annika Alica Specht, Julia Gesing, Roland Pfaeffle, Antje Koerner, Wieland Kiess

Institut

Hospital for Children and Adolescents, Leipzig University, Leipzig

Schlüsselwörter

Geschlechtsdysphorie, Transidentität, Komorbiditäten, geschlechtsangleichende Therapie

\section{Key words}

gender dysphoria, transgender, comorbidities, sex reassignment therapy

\section{Bibliografie}

DOI https://doi.org/10.1055/a-1066-4625

Online-Publikation: 17.12.2019

Klin Padiatr 2020; 232: 5-12

(c) Georg Thieme Verlag KG Stuttgart · New York

ISSN 0300-8630

Korrespondenzadresse

Prof. Dr. med. Wieland Kiess

Universitätsklinik und Poliklinik für Kinder

und Jugendliche Leipzig

Liebigstraße 20a

04103 Leipzig

Tel: 00493419726000 , Fax: 00493419726009

wieland.kiess@medizin.uni-leipzig.de

\section{ZUSAMMENFASSUNG}

Einleitung Die Geschlechtsdysphorie im Kindes- und Jugendalter (GD) zeigt sich durch eine Inkongruenz zwischen dem Geburtsgeschlecht und der gefühlten Geschlechtsidentität, welche in der Regel in der Pubertät zunimmt. Kinder und Jugendliche mit GD sind häufiger von psychischen Komorbiditäten wie Depressionen betroffen. Diese Arbeit soll einen Überblick über die von uns behandelten Patienten geben.

Methoden Es erfolgte eine Datenanalyse aus den Patientenakten von 66 Patienten, welche sich im Zeitraum von 2005 bis 2018 erstmals mit einer GD in unserer endokrinologischen Ambulanz vorstellten.

Ergebnisse Wir beobachteten einen Anstieg der Neuvorstellungen von maximal einem Patienten pro Jahr zwischen 2005 und 2011 auf zuletzt 18 Neuvorstellungen im Jahr 2018, wobe der Anteil an den insgesamt 14339 endokrinologischen Patienten desselben Zeitraums mit $0,49 \%$ gering ist. 54 der $\mathrm{Pa}$ tienten hatten ein weibliches und 12 ein männliches Geburtsgeschlecht, damit lag das Geschlechterverhältnis bei 4,5:1. Das Durchschnittsalter bei Erstvorstellung betrug 13,6 Jahre. 49 Patienten (74\%) überschritten das Pubertätsstadium P3/B3 bzw. P3/G3 nach Tanner. 48 (73\%) entwickelten erste Symptome einer GD vor der Pubertät und 30 (45\%) zeigten eine psychische Komorbidität. 15 Patienten ( $23 \%$ ) berichteten über negative Reaktionen ihres sozialen Umfelds nach ihrem Outing und 17 (26\%) machten Erfahrung mit Mobbing in der Schule. Schlussfolgerung Die Patientenzahl von Kindern und Jugendlichen mit GD ist in den letzten Jahren deutlich gestiegen. Zusätzlich zu der psychischen Belastung aufgrund der GD konnten wir bei ungefähr der Hälfte der Patienten typische psychische Komorbiditäten einer GD feststellen. Außerdem berichten Kinder und Jugendliche von negativen Reaktionen bezüglich ihre GD in der Schule und der Familie. Die hohe Variabilität des Verlaufs und der Komorbiditäten stellt die zentrale Herausforderung in der Therapie dar

\section{ABSTRACT}

Introduction Gender dysphoria (GD) in child hood and adolescence is characterized by an incongruence between sex at birth and gender identity, which usually increases during puberty. Gender dysphoric children and adolescents often suffer from psychological comorbidities such as depression. The purpose of our study is to give an overview of the patients treated in ou clinic.

Methods We analyzed data of 66 patients who presented with GD at our outpatient clinic between 2005 and 2018 .

Results We noted a rise of presentations with one or no patient with GD per year between 2005 and 2008 up to 18 patients in 2018, although the percentage of all 14.339 endocrinological outpatients (2005-2018) is low. 54 patients were assigned as female and 12 as male at birth resulting in a ratio of $4.5: 1$. The mean age at their first appointment was 13.6 years. 49 patients (74\%) had reached tanner stadium $\mathrm{P} 3 / \mathrm{B} 3$ or $\mathrm{P} 3 / \mathrm{G} 3$. $30(45 \%)$ showed symptoms of a comorbidity related to GD. $48(73 \%)$ showed symptoms of GD before puberty. 15 patients 
(23\%) experienced a negative response regarding their outing and $17(26 \%)$ were victims of mobbing in school.

Conclusion The number of patients increased in the last years.

About half of the patients suffered from a psychological comor- bidity. They often experienced negative response regarding their GD in the family. The variability of appearance and the comorbidities pose the challenge in the treatment of gender dysphoric children and adolescents.

\section{Einleitung}

Geschlechtsdysphorie (GD) im Kindes- und Jugendalter geht mit einem wachsenden Unbehagen des Kindes in Bezug auf das biologische Geschlecht einher. Charakteristisch ist besonders die zunehmende Ablehnung des biologischen Geschlechts mit Eintritt in die Pubertät, wobei Symptome oft schon in früher Kindheit auftreten. Die Diagnosekriterien der GD im Kindes- und Jugendalter sind dementsprechend ein anhaltendes und intensives Unbehagen bezüglich des biologischen Geschlechts, die Präferenz der typischen gegengeschlechtlichen Rollen oder die Verleugnung des eigenen Geschlechts, der präpubertäre Beginn und eine anhaltende Symptomatik von über 6 Monaten ( $\triangleright$ Tab. 1 ).

Studien zu dieser Thematik aus dem deutschsprachigen Raum sind rar. Es gibt lediglich einzelne Fallberichte [19] und Untersuchungen kleinerer Kohorten [4].

Für die Therapie von Kindern und Jugendlichen mit GD existiert eine S1-Leitlinie, die sich aktuell in Überarbeitung befindet [3]. Die aktuelle S3-Leitlinie zur Diagnostik, Beratung und Therapie von Geschlechtsinkongruenz, Geschlechtsdysphorie und Transgesundheit schließt Kinder und Jugendliche nicht mit ein. Die Therapie lehnt sich daher an Empfehlungen von Studien $[9,12,13]$ und der World Professional Association of Transgender Health's Standards of Care (WPATH-SOC) [10] an. Sie umfasst die Alltagstestphase, die Therapie mit GnRH-Analoga (reversibel), die Behandlung mit geschlechtsangleichenden Hormonen (teilweise reversibel) und die chirurgische Geschlechtsangleichung (zum großen Teil irreversibel) ( $>$ Abb. 1). Es sollte außerdem begleitend eine Psychotherapie angeboten werden bzw. erfolgen, da eine Geschlechtsdysphorie vermehrt mit psychiatrischen Komorbiditäten (Depressionen, selbstverletzendem Verhalten oder Suizidversuchen) $[15,17]$ einher gehen kann. Laut einer Studie von Wallien et al. [26] erfüllten $52 \%$ der Kinder und Jugendliche mit diagnostizierter GD mindestens eine andere Diagnose des DSM-III-R oder DSM-IV. Des Weiteren empfiehlt es sich, eine Familientherapie anzubieten. Eine Studie von Grossman und D'Augelli [17] zeigte, dass 35-73\% der Kinder und Jugendlichen gelegentlich bis häufig verbale Gewalt in Form von Beschimpfungen, Kritik, Bloßstellung vor Anderen und Beschuldigungen aufgrund ihrer Geschlechtsidentität durch ihre Eltern erlitten. 13-36\% der Befragten berichteten sogarvon regelmäßigen körperlichen Misshandlungen. Betroffene Kinder und Jugendliche begingen häufiger Suizidversuche.

Diese Arbeit soll einen Überblick über die Betreuung und Therapie, aber auch das soziale und familiäre Umfeld dieser noch sehr wenig betrachteten Patientengruppe geben. So soll ein Beitrag dazu geleistet werden, die Einschätzung und die klinische Versorgung von Kindern und Jugendlichen mit Geschlechtsdysphorie zu verbessern.

\section{Begriffsdefinitionen}

Im Folgenden werden verschiedene Begriffe zur Beschreibung des Geschlechts verwendet. Da sich die Sprache in einem ständigen Wandel befindet, werden die verwendeten Begriffe in ihrer Bedeutung zu Beginn klar definiert.

- Geschlechtsdysphorie (GD): Ein wachsendes Unbehagen in Bezug auf das biologische Geschlecht, früher als Geschlechtsidentitätsstörung (GID) bezeichnet, aber sowohl in der neuen S3-Leitlinie als auch dem WPATH zugunsten der GD abgeändert.

- Geschlechtsinkongruenz: Diskrepanz zwischen der Geschlechtsidentität bzw. dem gelebten/erlebten Geschlecht und den körperlichen Geschlechtsmerkmalen.

- Biologisches Geschlecht oder Geburtsgeschlecht: Das biologische Geschlecht bezeichnet das bei Geburt festgestellte Geschlecht (i.d.R. das chromosomale Geschlecht).

- Gelebtes/erlebtes Geschlecht: Das präferierte und/oder erlebte und gelebte Geschlecht, welches ein anderes oder alternatives Geschlecht zum biologischen Geschlecht darstellt.

\section{Methoden}

Zwischen 2005 und 2018 wurden 68 Kinder und Jugendliche mit Geschlechtsdysphorie in der endokrinologischen Ambulanz de Kinderklinik des Universitätsklinikums Leipzig erstmalig registriert. Hiervon schlossen wir 66 in unsere Analyse ein. Es wurden $2 \mathrm{~Pa}$ tienten ausgeschlossen, da diese lediglich einen Termin vereinbart hatten, zu diesem aber nicht erschienen waren. Alle Angaben zu Diagnosen und Untersuchungsergebnissen sowie demografische Daten der Kinder stammen aus den Patientenakten. Die Patientenakten beinhalten Arztbriefe, psychiatrische Diagnostik und besonders die durch die behandelnden Ärzte dokumentieren Gespräche mit Patienten und Eltern. Soziale Merkmale sowie medizinische Anamnese der Eltern, Informationen zu Frühsymptomen und dem Beginn der Geschlechtsdysphorie wurden den Anamnesen der Kinder und Eltern entnommen. Die Daten wurden nicht standardisiert erhoben und fehlenden Informationen nicht aktiv eingeholt. Die Studie beschränkt sich auf dokumentierte Daten und Fakten. Alle erhobenen Informationen wurden in einem Datenblatt zusammengetragen und anschließend anonymisiert deskriptiv analysiert. De Behandlungsvertrag am Universitätsklinikum Leipzig beinhaltet eine Einverständniserklärung zum anonymisierten Nutzen von Patientendaten. Für alle erfassten Patientinnen und Patienten liegt dieser Vertrag und das Einverständnis vor. Die Ethik-Kommission an der medizinischen Fakultät der Universität Leipzig erhob keine ethischen, wissenschaftlichen oder rechtlichen Bedenken gegen die anonymisierte Nutzung der Patientendaten im Rahmen dieser 
> Tab. 1 Diagnosekriterien bei Kindern sowie Jugendlichen und Erwachsenen mit Geschlechtsdysphorie nach DSM-V [2].

\begin{tabular}{|c|c|}
\hline Kinder & Jugendliche und Erwachsene \\
\hline $\begin{array}{l}\text { A. Eine deutliche Unstimmigkeit zwischen dem gelebten/erlebten } \\
\text { Geschlecht und dem biologischen Geschlecht über einen Zeitraum } \\
\text { von } 6 \text { Monaten hinweg, welche sich durch mindesten } 6 \text { der folgenden } \\
\text { Kriterien manifestiert (eines muss Kriterium A1 sein): }\end{array}$ & $\begin{array}{l}\text { A. Eine deutliche Unstimmigkeit zwischen dem gelebten/erlebten } \\
\text { Geschlecht und dem biologischen Geschlecht über einen Zeitraum von } \\
6 \text { Monaten hinweg, welche sich durch mindesten } 2 \text { der folgenden } \\
\text { Kriterien manifestiert: }\end{array}$ \\
\hline $\begin{array}{l}\text { 1. Starkes Unbehagen bezüglich des biologischen Geschlechts oder das } \\
\text { Bestehen darauf, dem anderen Geschlecht (oder einem alternativen } \\
\text { Geschlecht, das nicht dem biologischen Geschlecht entspricht) } \\
\text { anzugehören. }\end{array}$ & $\begin{array}{l}\text { 1. Starkes Unbehagen bezüglich des gelebten/erlebten Geschlechts und } \\
\text { dem primären und /oder sekundären Geschlechtsmerkmalen (oder bei } \\
\text { jungen Jugendlichen bezüglich der erwarteten sekundären Ge- } \\
\text { schlechtsmerkmale). }\end{array}$ \\
\hline $\begin{array}{l}\text { 2. Bei biologischen Jungen: deutliches Bevorzugen gegengeschlechtli- } \\
\text { cher und femininer Kleidung; bei biologischen Mädchen: deutliches } \\
\text { Bevorzugen maskuliner Kleidung und ein striktes Ablehnen typisch } \\
\text { femininer Kleidung. }\end{array}$ & $\begin{array}{l}\text { 2. Starker Wunsch, die primären und /oder sekundären Geschlechtsmerk- } \\
\text { male zu verlieren, da diese die Unstimmigkeit zu dem gelebten/ } \\
\text { erlebten Geschlecht (oder einem alternativen Geschlecht, das nicht } \\
\text { dem biologischen Geschlecht entspricht) hervorheben. }\end{array}$ \\
\hline $\begin{array}{l}\text { 3. Deutliches Präferieren gegengeschlechtlicher Rollen in Fantasiespie- } \\
\text { len und Rollenspielen. }\end{array}$ & $\begin{array}{l}\text { 3. Starker Wunsch, primäre und /oder sekundäre Geschlechtsmerkmale } \\
\text { des anderen Geschlechts zu haben. }\end{array}$ \\
\hline $\begin{array}{l}\text { 4. Deutliches Bevorzugen von Spielzeug, Spielen und Hobbies, welche } \\
\text { typischerweise dem anderen Geschlecht zugeordnet werden. }\end{array}$ & $\begin{array}{l}\text { 4. Starker Wunsch vom anderen Geschlecht (oder einem alternativen } \\
\text { Geschlecht, das nicht dem biologischen Geschlecht entspricht) zu sein. }\end{array}$ \\
\hline 5. Bevorzugen gegengeschlechtlicher Freunde. & $\begin{array}{l}\text { 5. Starker Wunsch, wie das andere Geschlecht (oder ein alternatives } \\
\text { Geschlecht, das nicht dem biologischen Geschlecht entspricht) } \\
\text { behandelt zu werden. }\end{array}$ \\
\hline $\begin{array}{l}\text { 6. Bei biologischen Jungen: eine starke Ablehnung typisch maskuliner } \\
\text { Spiele, Spielzeuge und Hobbies und das Vermeiden von Raufen und } \\
\text { Toben; bei biologischen Mädchen: eine starke Ablehnung typisch } \\
\text { femininer Spiele, Spielzeuge und Hobbies. }\end{array}$ & $\begin{array}{l}\text { 6. Überzeugung, dass man typische Gefühle hat und Verhaltensmuster } \\
\text { lebt wie das andere Geschlecht (oder ein alternatives Geschlecht, das } \\
\text { nicht dem biologischen Geschlecht entspricht). }\end{array}$ \\
\hline \multicolumn{2}{|l|}{ 7. Starke Ablehnung des eigenen biologischen Geschlechts. } \\
\hline \multicolumn{2}{|l|}{$\begin{array}{l}\text { 8. Starker Wunsch, dass die primären und /oder sekundären Ge- } \\
\text { schlechtsmerkmale zu dem gelebten/erlebten Geschlechts passen. }\end{array}$} \\
\hline $\begin{array}{l}\text { B. Das Kriterium muss mit einem signifikanten Stress und einer } \\
\text { Beeinträchtigung der Soziallebens, der Schule oder anderen wichtigen } \\
\text { Lebensbereichen einher gehen. }\end{array}$ & $\begin{array}{l}\text { B. Das Kriterium muss mit einem signifikanten Stress und einer Beeinträch- } \\
\text { tigung des Soziallebens, des Berufs oder anderen wichtigen Lebensbe- } \\
\text { reichen einher gehen. }\end{array}$ \\
\hline
\end{tabular}

Studie (Reg.-Nr.: 044/2003). Die Studie entstand im Rahmen eine Promotionsarbeit der medizinischen Fakultät der Universität Leipzig (A. Specht).

\section{Ergebnisse}

\section{Alter bei Erstsymptomen, Alter bei Erstvorstellung} Diagnostik, Diagnose

Wir beobachteten einen Anstieg der Anzahl an Patienten, die sich aufgrund einer GD in der Sprechstunde vorstellten. Von 2005 bis einschließlich 2016 stellten sich 36 Kinder und Jugendliche (54,5\%) in der Sprechstunde vor. In den Jahren 2017 und 2018 stellten sich mit insgesamt 30 Kindern und Jugendlichen 4,6-mal so viele $\mathrm{Pa}$ tienten pro Jahr vor ( $\triangleright$ Abb. 2). Gleichzeitig ist der Anteil der Pa tienten mit GD an den Patienten der endokrinologischen Ambulanz über die gesamte Zeit mit ca. 0,49\% gering. Bei 66 Kindern bzw. Jugendlichen wurden eine Variante der geschlechtlichen Entwicklung, Chromosomenanomalien oder metabolische Ursachen für eine Geschlechtsdysphorie ausgeschlossen. Andere Psychopathologien, welche eine primäre Behandlung erfordern, wurden vor Beginn einer Hormontherapie durch die betreuenden Psychologen/Psychiater ausgeschlossen. Von diesen 66 Patienten waren 12 biologisch männlich und 54 biologisch weiblich. Das entsprach einem Verhältnis von ca. 4,5:1 (w:m). Als Frühsymptome der Geschlechtsdysphorie berichteten Kinder und Eltern die Ablehnung geschlechtsspezifischen Verhaltens, Kleidung und Spielzeugs, vorrangig Freunde des anderen Geschlechts, Ablehnung des eigenen Geschlechts und Körpers sowie den Wunsch, das Geschlecht im Erwachsenenalter zu ändern ( $\triangleright$ Tab. 2). Bei 48 Kindern $(72,7 \%$ ) traten diese Frühsymptome vor der Pubertät auf.16 Kinder (24,2\%) entwickelten erste Symptome mit Eintritt in die Pubertät bzw. im Verlauf der Pubertät.

Die Kinder bzw. Jugendlichen waren bei Erstvorstellung in der endokrinologischen Ambulanz im Durchschnitt 13,6 ]ahre alt ([4 $17], s=2,82) .44$ der 54 Mädchen $(81,5 \%)$ hatten bei Erstvorstellung bereits ihre Menarche. Im Durchschnitt vergingen 2,2 Jahre zwischen der Menarche und der ersten Vorstellung in der kinderendokrinologischen Ambulanz. Zum Zeitpunkt der Erstvorstellung hatten 49 Jugendliche (74,2 \%) das Tanner-Stadium P3/B3 bzw. P3/ G3 überschritten ( $\triangleright$ Tab. 3 ).

\section{Familie, Freunde und Schule}

Die Eltern von 27 Kindern (40,9\%) lebten zusammen, während 20 Elternpare (30,3\%) dauerhaft getrennt lebten. Im Falle der getrenntlebenden Eltern hatte in der Hälfte der Fälle die Mutter das alleinige Sorgerecht. Fünf Kinder (7,6\%) wohnten dauerhaft oder vorübergehend bei einer Pflegefamilie. 35 Patienten $(53,0 \%$ ) gaben an, nach dem Outing Unterstützung von beiden Elternteilen erfahren zu haben, 16 (24,2\%) Jugendliche berichteten über negative Reaktionen von mindestens einem Familienmitglied. 
> Tab. 1 Diagnosekriterien bei Kindern sowie Jugendlichen und Erwachsenen mit Geschlechtsdysphorie nach DSM-V [2].

\begin{tabular}{|c|c|}
\hline Kinder & Jugendliche und Erwachsene \\
\hline $\begin{array}{l}\text { A. Eine deutliche Unstimmigkeit zwischen dem gelebten/erlebten } \\
\text { Geschlecht und dem biologischen Geschlecht über einen Zeitraum } \\
\text { von } 6 \text { Monaten hinweg, welche sich durch mindesten } 6 \text { der folgenden } \\
\text { Kriterien manifestiert (eines muss Kriterium A1 sein): }\end{array}$ & $\begin{array}{l}\text { A. Eine deutliche Unstimmigkeit zwischen dem gelebten/erlebten } \\
\text { Geschlecht und dem biologischen Geschlecht über einen Zeitraum von } \\
6 \text { Monaten hinweg, welche sich durch mindesten } 2 \text { der folgenden } \\
\text { Kriterien manifestiert: }\end{array}$ \\
\hline $\begin{array}{l}\text { 1. Starkes Unbehagen bezüglich des biologischen Geschlechts oder das } \\
\text { Bestehen darauf, dem anderen Geschlecht (oder einem alternativen } \\
\text { Geschlecht, das nicht dem biologischen Geschlecht entspricht) } \\
\text { anzugehören. }\end{array}$ & $\begin{array}{l}\text { 1. Starkes Unbehagen bezüglich des gelebten/erlebten Geschlechts und } \\
\text { dem primären und /oder sekundären Geschlechtsmerkmalen (oder bei } \\
\text { jungen Jugendlichen bezüglich der erwarteten sekundären Ge- } \\
\text { schlechtsmerkmale). }\end{array}$ \\
\hline $\begin{array}{l}\text { 2. Bei biologischen Jungen: deutliches Bevorzugen gegengeschlechtli- } \\
\text { cher und femininer Kleidung; bei biologischen Mädchen: deutliches } \\
\text { Bevorzugen maskuliner Kleidung und ein striktes Ablehnen typisch } \\
\text { femininer Kleidung. }\end{array}$ & $\begin{array}{l}\text { 2. Starker Wunsch, die primären und /oder sekundären Geschlechtsmerk- } \\
\text { male zu verlieren, da diese die Unstimmigkeit zu dem gelebten/ } \\
\text { erlebten Geschlecht (oder einem alternativen Geschlecht, das nicht } \\
\text { dem biologischen Geschlecht entspricht) hervorheben. }\end{array}$ \\
\hline $\begin{array}{l}\text { 3. Deutliches Präferieren gegengeschlechtlicher Rollen in Fantasiespie- } \\
\text { len und Rollenspielen. }\end{array}$ & $\begin{array}{l}\text { 3. Starker Wunsch, primäre und /oder sekundäre Geschlechtsmerkmale } \\
\text { des anderen Geschlechts zu haben. }\end{array}$ \\
\hline $\begin{array}{l}\text { 4. Deutliches Bevorzugen von Spielzeug, Spielen und Hobbies, welche } \\
\text { typischerweise dem anderen Geschlecht zugeordnet werden. }\end{array}$ & $\begin{array}{l}\text { 4. Starker Wunsch vom anderen Geschlecht (oder einem alternativen } \\
\text { Geschlecht, das nicht dem biologischen Geschlecht entspricht) zu sein. }\end{array}$ \\
\hline 5. Bevorzugen gegengeschlechtlicher Freunde. & $\begin{array}{l}\text { 5. Starker Wunsch, wie das andere Geschlecht (oder ein alternatives } \\
\text { Geschlecht, das nicht dem biologischen Geschlecht entspricht) } \\
\text { behandelt zu werden. }\end{array}$ \\
\hline $\begin{array}{l}\text { 6. Bei biologischen Jungen: eine starke Ablehnung typisch maskuliner } \\
\text { Spiele, Spielzeuge und Hobbies und das Vermeiden von Raufen und } \\
\text { Toben; bei biologischen Mädchen: eine starke Ablehnung typisch } \\
\text { femininer Spiele, Spielzeuge und Hobbies. }\end{array}$ & $\begin{array}{l}\text { 6. Überzeugung, dass man typische Gefühle hat und Verhaltensmuster } \\
\text { lebt wie das andere Geschlecht (oder ein alternatives Geschlecht, das } \\
\text { nicht dem biologischen Geschlecht entspricht). }\end{array}$ \\
\hline \multicolumn{2}{|l|}{ 7. Starke Ablehnung des eigenen biologischen Geschlechts. } \\
\hline \multicolumn{2}{|l|}{$\begin{array}{l}\text { 8. Starker Wunsch, dass die primären und /oder sekundären Ge- } \\
\text { schlechtsmerkmale zu dem gelebten/erlebten Geschlechts passen. }\end{array}$} \\
\hline $\begin{array}{l}\text { B. Das Kriterium muss mit einem signifikanten Stress und einer } \\
\text { Beeinträchtigung der Soziallebens, der Schule oder anderen wichtigen } \\
\text { Lebensbereichen einher gehen. }\end{array}$ & $\begin{array}{l}\text { B. Das Kriterium muss mit einem signifikanten Stress und einer Beeinträch- } \\
\text { tigung des Soziallebens, des Berufs oder anderen wichtigen Lebensbe- } \\
\text { reichen einher gehen. }\end{array}$ \\
\hline
\end{tabular}

Studie (Reg.-Nr.: 044/2003). Die Studie entstand im Rahmen eine Promotionsarbeit der medizinischen Fakultät der Universität Leipzig (A. Specht).

\section{Ergebnisse}

\section{Alter bei Erstsymptomen, Alter bei Erstvorstellung} Diagnostik, Diagnose

Wir beobachteten einen Anstieg der Anzahl an Patienten, die sich aufgrund einer GD in der Sprechstunde vorstellten. Von 2005 bis einschließlich 2016 stellten sich 36 Kinder und Jugendliche (54,5\%) in der Sprechstunde vor. In den Jahren 2017 und 2018 stellten sich mit insgesamt 30 Kindern und Jugendlichen 4,6-mal so viele $\mathrm{Pa}$ tienten pro Jahr vor ( $\triangleright$ Abb. 2). Gleichzeitig ist der Anteil der Pa tienten mit GD an den Patienten der endokrinologischen Ambulanz über die gesamte Zeit mit ca. 0,49\% gering. Bei 66 Kindern bzw. Jugendlichen wurden eine Variante der geschlechtlichen Entwicklung, Chromosomenanomalien oder metabolische Ursachen für eine Geschlechtsdysphorie ausgeschlossen. Andere Psychopathologien, welche eine primäre Behandlung erfordern, wurden vor Beginn einer Hormontherapie durch die betreuenden Psychologen/Psychiater ausgeschlossen. Von diesen 66 Patienten waren 12 biologisch männlich und 54 biologisch weiblich. Das entsprach einem Verhältnis von ca. 4,5:1 (w:m). Als Frühsymptome der Geschlechtsdysphorie berichteten Kinder und Eltern die Ablehnung geschlechtsspezifischen Verhaltens, Kleidung und Spielzeugs, vorrangig Freunde des anderen Geschlechts, Ablehnung des eigenen Geschlechts und Körpers sowie den Wunsch, das Geschlecht im Erwachsenenalter zu ändern ( $\triangleright$ Tab. 2). Bei 48 Kindern $(72,7 \%$ ) traten diese Frühsymptome vor der Pubertät auf.16 Kinder (24,2\%) entwickelten erste Symptome mit Eintritt in die Pubertät bzw. im Verlauf der Pubertät.

Die Kinder bzw. Jugendlichen waren bei Erstvorstellung in der endokrinologischen Ambulanz im Durchschnitt 13,6 ]ahre alt ([4 $17], s=2,82) .44$ der 54 Mädchen $(81,5 \%)$ hatten bei Erstvorstellung bereits ihre Menarche. Im Durchschnitt vergingen 2,2 Jahre zwischen der Menarche und der ersten Vorstellung in der kinderendokrinologischen Ambulanz. Zum Zeitpunkt der Erstvorstellung hatten 49 Jugendliche (74,2 \%) das Tanner-Stadium P3/B3 bzw. P3/ G3 überschritten ( $\triangleright$ Tab. 3 ).

\section{Familie, Freunde und Schule}

Die Eltern von 27 Kindern (40,9\%) lebten zusammen, während 20 Elternpare (30,3\%) dauerhaft getrennt lebten. Im Falle der getrenntlebenden Eltern hatte in der Hälfte der Fälle die Mutter das alleinige Sorgerecht. Fünf Kinder (7,6\%) wohnten dauerhaft oder vorübergehend bei einer Pflegefamilie. 35 Patienten $(53,0 \%$ ) gaben an, nach dem Outing Unterstützung von beiden Elternteilen erfahren zu haben, 16 (24,2\%) Jugendliche berichteten über negative Reaktionen von mindestens einem Familienmitglied. 


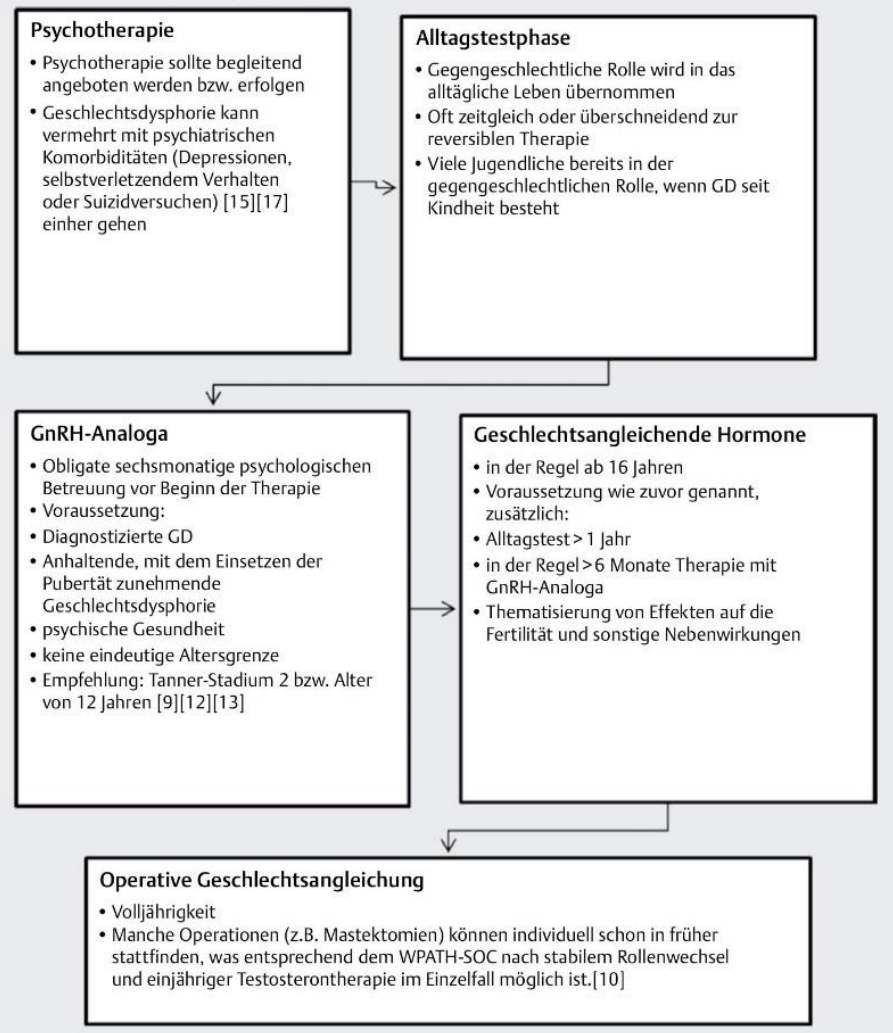

- Abb. 1 Behandlungsschema bei Kindern und Jugendlichen mit Geschlechtsdysphorie.

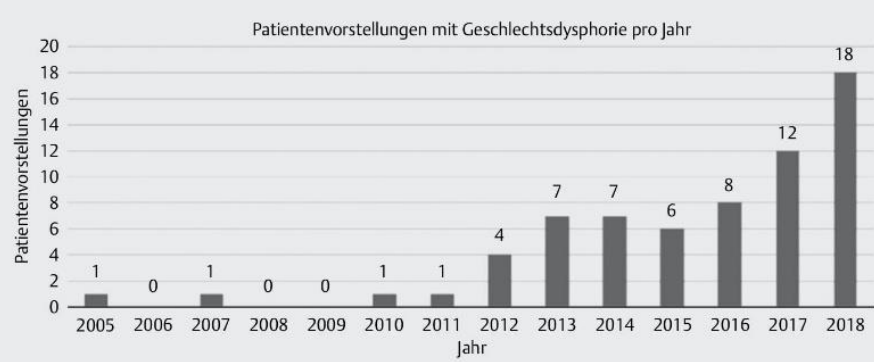

- Abb. 2 Patientenvorstellungen mit Geschlechtsdysphorie pro Jahr. 
> Tab. 2 Charakteristika der Kohorte.

\begin{tabular}{|l|l|l|l|}
\hline Klinische Daten & & & \\
\hline & Alle $(\mathrm{n}=66)$ & FtM $(\mathrm{n}=54)$ & MtF ( $\mathrm{n}=12)$ \\
\hline Alter bei Erstvorstellung & & & \\
\hline Durchschnitt (Minimum/Maximum) in Jahren & $13,61(4 / 17)$ & $13,87(7 / 17)$ & $12,42(4 / 17)$ \\
\hline Tanner-Stadium bei Erstvorstellung & & & \\
\hline P 1-3 & 14 & 9 & 5 \\
\hline P >3 & 49 & 44 & 5 \\
\hline B 1-3 & & 11 & \\
\hline B >3 & & 41 & 5 \\
\hline G 1-3 & & & 5 \\
\hline G >3 & & & \\
\hline Menarche & & $12,37(9 / 16)$ & \\
\hline Durchschnitt (Minimum/Maximum) in Jahren & & $2,22(0 / 5)$ & \\
\hline Zeit von Menarche bis Erstvorstellung in Jahren & & & 7 \\
\hline Therapie & 42 & 35 & 3 \\
\hline Therapie mit GnRH-Analoga & 24 & 5 & 0 \\
\hline Therapie mit gegengeschlechtlichen Hormonen & 5 & & \\
\hline Geschlechtsangleichende Operationen & & \\
\hline
\end{tabular}

- Tab. 3 Anzeichen der Geschlechtsdysphorie und psychiatrische Komorbiditäten (Mehrfachnennungen möglich).

\begin{tabular}{|l|c|}
\hline & Alle (n=66) \\
\hline Anzeichen der Geschlechts dysphorie & $52(78,8)$ \\
\hline $\begin{array}{l}\text { Verhaltensweisen, Freunde und stereotypes Spielzeug } \\
\text { des anderen Geschlechts (\%) }\end{array}$ & $33(50,0)$ \\
\hline $\begin{array}{l}\text { Ablehnung geschlechtsspezifischer Kleidung oder } \\
\text { Rollen (\%) }\end{array}$ & $19(28,8)$ \\
\hline $\begin{array}{l}\text { Ablehnung des eigenen Geschlechts/der } \\
\text { Geschlechtsorgane (\%) }\end{array}$ & $2(3,0)$ \\
\hline $\begin{array}{l}\text { Wunsch/Überzeugung, das Geschlecht wird sich } \\
\text { ändern (\%) }\end{array}$ & $5(7,5)$ \\
\hline Psychiatrische Komorbiditäten & $14(21,2)$ \\
\hline Essstörung (\%) & $16(24,2)$ \\
\hline Negative Selbstbild (\%) & $7(10,6)$ \\
\hline Selbstverletzendes Verhalten (\%) & $14(21,2)$ \\
\hline Angststörung (\%) & $9(13,9)$ \\
\hline Depressive Episoden (\%) & $1(1,5)$ \\
\hline Suizidgedanken (\%) & \\
\hline Suizidversuch (\%) & \\
\hline
\end{tabular}

Es outeten sich bereits 57 Jugendliche $(86,4 \%)$ vor ihrer Schulklasse und 55 Jugendliche $(83,3 \%)$ lebten dauerhaft in ihrer präferierten Geschlechterrolle. Von ihnen gaben 40 Jugendliche $(70,2 \%)$ an, von ihren Klassenkameraden und Freunden unterstützt worden zu sein, während 7 Jugendliche (12,3\%) von gemischten oder überwiegend ablehnenden Reaktionen erzählten. 50 Jugendliche $(75,8 \%)$ berichteten, Freundschaften zu haben, während 6 Jugendliche $(9,1 \%)$ angaben, keine engen Freunde zu haben. Von Mob bing und Ausgrenzung, die auf die Geschlechtsdysphorie zurückgeführt wurde, berichteten 17 Jugendliche (25,8\%). Zehn Kinder
(15,2\%) beschrieben sich als Außenseiter in der Schule und 17 $(25,8 \%)$ wechselten außerplanmäßig die Schule.

\section{Behandlung, Komorbiditäten und Psychotherapie}

Aktuell werden 53 Kinder bzw. Jugendliche $(80,3 \%$ ) durch die Klinik und Poliklinik für Kinder- und Jugendmedizin Leipzig oder eine andere Klinik oder Praxis betreut. 12 Jugendliche (18,2\%) sind ohne Angabe von Gründen nicht mehr in der Sprechstunde erschienen und ein Patient (1,5\%) hat die Behandlung abgebrochen. 58 Patienten $(87,9 \%)$ sind oder waren regelmäßig in psychologischer Betreuung. Bei 30 dieser Jugendlichen $(51,7 \%$ ) ging aus der psychologischen differenzialdiagnostischen Untersuchung und der Anamnese hervor, dass mindestens eine der für Kinder mit Geschlechtsdysphorie typischen Komorbiditäten bestand. Am häufigsten wurde selbstverletzendes Verhalten, ein negatives Selbstbild und Depressionen angegeben, weitere Komorbiditäten waren Selbstmordgedanken, Angststörungen und Essstörungen ( $\triangleright$ Tab. 2 ). Drei Kinder (4,5\%) gaben an körperlichen Missbrauch erfahren zu haben. Von den Kindern und Jugendlichen, die sich mit einem Tanner-Stadium P/B/G $>3$ erstmalig vorstellten, litten 26 (53\%) an mindestens einer Komorbidität, wohingegen von den Kindern und Jugendlichen mit einen Stadium P/B/G 1-3 lediglich 2 (14,3\%) von Komorbiditäten betroffen waren. 42 Patienten (63,6\%) wurden mit GnRH-Analoga behandelt, 24 dieser Patienten (36,4\% aller Patienten) nahmen geschlechtsangleichende Hormone ein. Operationen zur Geschlechtsangleichung wurden bei 5 der 66 Patienten (7,6\%) durchgeführt ( $\triangleright$ Tab. 3). Die durchgeführten Operationen waren 4 Mastektomien und eine Entfernung der weiblichen Geschlechtsorgane mit Mastektomie. Die Voraussetzungen für diese Eingriffe waren neben der diagnostizierten GD und dem Wunsch der Patienten in 4 Fällen die Volljährigkeit. In einem Fall wurde die Ablatio mammae in dem Alter von 17 Jahren durch geführt. 


\section{Diskussion}

\section{Alter bei Erstsymptomen, Alter bei Erstvorstellung, Diagnostik, Diagnose}

Der Anteil der Kinder und Jugendlichen mit GD an den gesamten Patienten der endokrinologischen Ambulanz ist mit $0,46 \%$ gering. Wir stellen einen deutlichen Anstieg der Patientenzahl in den letzten 2 Jahren fest. Dies entspricht auch einer neuen Studie aus Amsterdam, in der ebenfalls ein starker Anstieg der Patentenzahlen beobachtet wurde [28]. Das Geschlechterverhältnis in unserer Studie widerspricht den Geschlechterverhältnissen in anderen Studien. Das Geschlechterverhältnis ausgehend vom Geburtsgeschlecht von Jungen zu Mädchen mit GD ist laut Literatur präpubertär weit zugunsten der Jungen verschoben (ca. 16:1) [6], wobei es sich im Laufe der Pubertät nahezu vollständig ausgleicht $[6,8,24,29]$. Eine mögliche Ursache könnte eine höhere gesellschaftliche Akzeptanz bzw. Toleranz von jungenhaften Eigenschaften und Verhaltensweisen bei Mädchen als mädchenhaften Eigenschaften und Verhaltensweisen bei]ungen sein $[5,27,30]$. Des Weiteren legen Studien von Behandlungszentren aus Toronto und Amsterdam nahe, dass sich das Geschlechterverhältnis über die letzten Jahre bzw. Jahrzehnte geändert hat: Während in der Zeit vor 2000 das Geschlechterverhältnis auf das Geburtsgeschlecht bezogen von Jungen zu Mädchen mit GD in Toronto bei 5,75:1 und in Amsterdam bei 2,93:1 [8] lag, näherte es sich nach 2000 in Toronto auf 3,41:1 [29] und in Amsterdam auf 1,68:1 (2008-2011) [24] an. Diese Theorie wird durch eine deutsche Studie von Garrels et al. [16] und anderen europäischen Studien $[14,21,25]$ bestärkt. Neuste Untersuchungen sprechen gar von einer Umkehr des Geschlechterverhältnisses $[7,23]$. Obwohl in unserer Studie das Geschlechterverhältnis zugunsten der Mädchen verschoben ist, gleicht es sich in den letzten Jahren doch deutlich an und entspricht so den Tendenzen neuerer Studien [24, 29]. Im Zeitraum vom 2005 bis 2016 liegt die Verteilung von Patienten mit weiblichem zu männlichem Geburtsgeschlecht bei 7,2:1, wohingegen sich das Verhältnis zwischen 2017 und 2018 auf 3,3:1 annähert. Jungen sind im Durchschnitt bei der Erstvorstellung jünger (12,4 Jahre) als Mädchen (13,8 Jahre). Die zunehmende mediale Aufmerksamkeit für die Geschlechtsdysphorie im Kindes- und Jugendalter und die verbesserten Informationsmöglichkeiten könnten dazu beitragen, dass Jugendliche sich früher in der Sprechstunde vorstellen. Nichtsdestotrotz scheint die Verantwortung, eine GD zu erkennen und den Erstkontakt zu spezialisierten Zentren herzustellen, zurzeit noch bei den niedergelassenen Pädiatern zu liegen.

Ein durchschnittliches Alter bei Erstvorstellung von 13,6 Jahren und eine dementsprechend fortgeschrittene körperliche Entwicklung kann Ärzte und Patienten vor Probleme stellen. Die WPATHSOC [10] empfehlen eine frühe Therapie mit GnRH-Analoga (ab Tanner-Stadium 2). Studien zeigten, dass das psychische und operative Ergebnis besser ist, wenn die Pubertät bei Behandlungsbeginn noch nicht zu weit vorangeschritten ist $[9,13]$. Um eine den Standards entsprechende Behandlung der Jugendlichen leisten zu können, ist es notwendig, dass Kinder bzw. Jugendliche und deren Eltern sich früh an spezialisierte Behandlungszentren wenden. So ist es möglich, die wachsenden Geschlechtsdys phorie während der Pubertät und Probleme innerhalb der Familie oder dem Freundes- kreis frühzeitig zu erkennen sowie Therapieentscheidungen ohne zeitlichen Druck zu treffen.

\section{Familie, Freunde und Schule}

Mit 30,3\% leben verglichen mit der Studie von Steensma [24] weniger Kinder in einem Haushalt mit beiden Elternteilen zusammen. Das Sorgerecht für die Kinder, deren Eltern geschieden sind, liegt in $50 \%$ der Fälle bei den Müttern, die übrigen getrennt lebenden Eltern teilen sich das Sorgerecht. Die familiären Verhältnisse und auch die Reaktionen der Eltern auf ein Coming-Out ihrer Kinder legen nahe, dass eine Familientherapie wichtige Unterstützung darin bieten kann, Alltagsprobleme gemeinsam zu lösen und zu vermitteln. So haben insgesamt 16 Jugendliche $(24,2 \%)$ negative Reaktionen in Bezug auf ihre Geschlechtsdysphorie innerhalb der eigenen Familie erfahren. Die Jugendlichen berichteten von Ablehnung eines oder mehrerer Familienmitglieder. Erfahrungen mit körperlicher Misshandlung wurde von 3 Kindern (4,5\%) berichtet, wobei aus der Anamnese nicht hervorgeht, in welchem Zusammenhang diese zur Geschlechtsdysphorie steht. Auch andere Studien haben gezeigt, dass Kinder und Jugendliche mit GD häufiger Opfer verbaler und körperlicher Gewalt werden und diese sich negativ auf die psychische Gesundheit auswirkt $[1,17]$.

55 Jugendlichen $(83,3 \%$ ) leben bereits in der präferierten Geschlechterrolle. Häufig haben die Jugendlichen positive Reaktionen nach ihrem Outing erhalten, jedoch berichten 17 Jugendliche $(25,8 \%)$ von Mobbing aufgrund ihrer Geschlechtsdysphorie. Studien zeigen, dass Mobbing ein niedriges Selbstbewusstsein, Einsamkeit, Depressionen, Suizidgedanken und -versuche und Suizide hervorrufen kann [22] Diese Erfahrungen können die weitere Schulzeit bis hin ins Erwachsenenalter beeinflussen [18] und sollten daher im ärztlichen Gespräch thematisiert werden.

Behandlung, Komorbiditäten und Psychotherapie

Die Hemmung der Hypophysen-Gonadenachse mittels GnRH-Analoga entspricht nach dem Einsetzen der Pubertät (mindestens Tanner-Stadium B2/G2) den aktuellen Leitlinien. Von den dargestellten Patienten werden 42 (63,6\%) mit GnRH-Analoga behandelt, 24 dieser Patienten ( $36,4 \%$ aller Patienten) nehmen geschlechtsangleichende Hormone ein. Operationen zur Geschlechtsangleichung wurden bei 5 der 66 Patienten (7,6\%) durchgeführt was entsprechend dem WPATH-SOC nach stabilem Rollenwechsel und einjäh riger Testosterontherapie im Einzelfall möglich ist [10]. Da sich unsere Analyse nur auf die Daten der Jahre 2006-2018 beziehen, können wir noch keine Aussage über das längerfristige Fortbestehen der Geschlechtsdysphorie bei unseren Patienten machen. Die Therapie mit GnRH-Analoga und geschlechtsangleichenden Hormo nen wurde nur von einem Patienten unterbrochen. Die übrigen 41 Patienten führten die Therapie nach Beginn weiter, was eine $\mathrm{Zu}$ friedenheit mit der Unterdrückung der Pubertät vermuten lässt. Obwohl die Behandlung mit GnRH-Analoga bei Jugendlichen ab Erreichen des Tanner-Stadiums 2 laut der S1-Leitlinie [3], den WPATHSOC [10] und den internationalen Leitlinien [9] leitliniengerecht ist, wird sie aufgrund möglicher Nebenwirkungen und dem Eingriff in den sensiblen Hormonhaushalt während der Pubertät kontrovers diskutiert. Erste Langzeitstudien [13] und eine Fallbericht [9] konnten aber keine negativen Auswirkungen auf die geistige und körperliche Entwicklung des Jugendlichen feststellen und neue Stu- 
dien belegen ein positives Outcome der psychischen Gesundheit durch eine Therapie mit GnRH-Analoga [11]. Kritiker der Therapie mit GnRH-Analoga und geschlechtsangleichenden Hormonen im frühen Jugendalter weisen auf die bisher nicht oder nur unzureichend erforschten Auswirkungen der Medikamente auf die psychosexuelle Entwicklung des Kindes hin [20]. 58 der Jugendlichen $(87,9 \%)$ waren in regelmäßiger psychologischer Betreuung. Wir fanden aufgrund der psychologischen differenzialdiagnostischen Untersuchung und der Anamnesen heraus, dass 30 Jugendliche (45,5\%) Anzeichen mindestens einer psychischen Komorbidität auswiesen. Die Häufigkeit der psychischen Komorbiditäten und die gestellten Diagnosen decken sich mit den Ergebnissen andere Studien $[15,17]$. Kinder und Jugendliche, bei denen die Pubertätsentwicklung bei Erstvorstellung bereits über das Stadium P3/G3/B nach Tanner überschritten war, leiden häufiger unter Komorbiditäten als Kinder und Jugendliche, die sich früher vorgestellt haben und dementsprechend früher therapiert werden konnten. Zu be achten ist allerdings, dass nur schwer zu trennen ist, welchen Ursprung die psychischen Komorbiditäten haben. Daher ist davon auszugehen, dass einige Komorbiditäten ebenso aus eigener Unsicherheit und sekundären Interaktionsproblemen mit dem familiären und sozialen Umfeld resultieren können. Eine weitere Limitation der Aussage ergibt sich aus nicht standardisiert dokumentierten Austausch zwischen behandelnden Psychologen und Pädiatern. Der Zugang zu Patienteninformationen bezüglich psy chologischer und psychiatrischer Behandlungen ist begrenzt. Jedoch sollten neue Möglichkeiten geprüft werden, die Kommunikation zwischen den betreuenden Ärzten zu verbessern, um die Therapie der Kinder und Jugendlichen mit Geschlechtsdysphorie zu optimieren. Eine Möglichkeit, die in einigen deutschen Behand lungszentren eingeführt wurde, ist eine interdisziplinäre Sprech stunde bestehend aus Psychologen bzw. Kinder- und Jugendpsychiatern, Pädiatern und wahlweise Ärzte aus anderen Fachrichtungen, die die Behandlung der Jugendlichen ergänzen.

\section{Schlussfolgerung}

Zusammenfassend stehen trotz der Limitationen der Studie wichtige Aussagen im Mittelpunkt: Durch einen deutlichen Anstieg der Patientenzahlen besonders in den letzten Jahren gewinnt die Geschlechtsdysphorie im Kindes- und Jugendalter in unserer endokrinologischen Sprechstunde an Bedeutung. Die Kinder und Jugendlichen stellen sich oft erst nach Pubertätseintritt vor, sodass die Ausbildung sekundärer Geschlechtsmerkmale bereits vorangeschritten ist. Wir haben festgestellt, dass Kinder und Jugendliche mit GD an verschiedenen psychischen Komorbiditäten leiden. Zusammen mit negativen Reaktionen auf das Outing in der Schule oder auch der Familie bedeutet das eine zusätzliche Belastung zu der bestehenden GD. Daher is eine verstärkte Zusammenarbeit aller behandelnden Ärzte wünschenswert. Besonders familiäre Konflikte und Probleme im sozialen Umfeld können frühzeitig erkannt werden. Eine interdisziplinäre Spezialsprechstunde aus Pädiatern, Psychologen und ggf. anderen Fachrichtungen wie der Endokrinologie, Urologie oder Gynäkologie kann eine Signalwirkung für Jugendliche und auch niedergelassene Pädiater haben, sich selbst bzw. den Patienten früher über spezialisierte Zentren zu informieren. So kann ein rechtzeitiger Beginn psychologischer Unterstützung realisiert und mögliche Therapien ohne Zeitdruck und mit gewissenhaftem Abwägen begonnen werden.

\section{Stellungsnahme zur Autorenschaft}

A. Specht: contribution to study concept and design + acquisition of data + analysis and interpretation of data; J.Gesing: contribution to study concept and design + revising manuscript; R. Pfäffle: revising manuscript; A. Körner: revising manuscript; W. Kiess: contribution to study concept and design + analysis and interpretation of data.

Interessenkonflikt

Die Autoren erklären, dass keine Interessenkonflikte bestehen.

Literatur

[1] Almeida J, Johnson RM, Corliss HL et al. Emotional distress among LGBT youth: The influence of perceived discrimination based on sexual orientation. J Youth Adolesc 2009; 38: 1001-1014

[2] American Psychiatric Association Diagnostic and statistical manual of mental disorders (DSM-5 ${ }^{\circledR}$ ). American Psychiatric Pub; 2013

[3] Arbeitsgemeinschaft der Wissenschaftlichen Medizinischen Fachgemeinschaften (AWMF)-Ständige Kommision der Leitlinien. AWMFRegister "Leitlinien" Registernummer 028 - 014; Stand: 01.08.2013 (in Uberarbeitung), gültig bis 01.08.2018, verfügbar: https://www. awmf.org/leitlinien/detail/I/028-014.html; Zugriff am 17.06.2019

[4] Becker I, Gjergji-Lama V, Romer G et al. Merkmale von Kindern und Jugendlichen mit Geschlechtsdysphorie in der Hamburger Spezialsprechstunde. Prax Kinderpsychol. Kinderpsychiatr 2014; 63: 486-509

[5] Blakemore JEO. Children's beliefs about violating gender norms: Boys shouldn't look like girls, and girls shouldn't act like boys. Sex Roles 2003; 48: 411-419

[6] Bosinski HA, Arndt R, Sippell WG et al. Geschlechtsidentitätsstörungen bei Kindern und Jugendlichen Nosologie und Epidemiologie. Monatsschr Kinderheilkd 1996; 144: 1235-1241

[7] Carmichael P, Steensma TD, Zucker K]. Evidence for a Change in the Sex Ratio of Children Referred for Gender Dysphoria: Data From the Gender Identity Development Service in London (2000-2017). I Sex Med 2018; 15: 1381-1383

[8] Cohen-Kettenis PT, Owen A, Kaijser VG et al. Demographic characteristics, social competence, and behavior problems in children with gender identity disorder: A cross-national, cross-clinic comparative analysis. J Abnorm Child Psychol 2003; 31: 41-53

[9] Cohen-Kettenis PT, Schagen SE, Steensma TD et al. Puberty suppression in a gender-dysphoric adolescent: A 22-year follow-up. Arch Sex Behav 2011; 40: 843-847

[10] Coleman E, Bockting W, Botzer M et al. Standards of care for the health of transsexual, transgender, and gender-nonconforming people, version 7. Int J Transgenderism 2012; 13: 165-232

[11] De Vries AL, McGuire JK, Steensma TD et al. Young adult psychological outcome after puberty suppression and gender reassignment. Pediatrics 2014; 134 (4): 696-704

[12] De Vries AL, Steensma TD, Doreleijers TA et al. Puberty suppression in adolescents with gender identity disorder: A prospective follow-up study. J Sex Med 2011; 8: 2276-2283 
[13] Delemarre-van de Waal HA, Cohen-Kettenis PT. Clinical management of gender identity disorder in adolescents: A protocol on psychological and paediatric endocrinology aspects. Eur J Endocrinol 2006; 155: $131-137$

[14] Dixen JM, Maddever $\mathrm{H}$, Van Maasdam J et al. Psychosocial characteristics of applicants evaluated for surgical gender reassignment. Arch Sex Behav 1984; 13: 269-276

[15] Fitzpatrick KK, Euton SJ, Jones JN et al. Gender role, sexual orientation and suicide risk. J Affect Disord 2005; 87: 35-42

[16] Garrels L, Kockott G, Michael N et al. Sex ratio of transsexuals in Germany: The development over three decades. Acta Psychiatr Scand 2000; 102: 445-448

[17] Grossman AH, D'augelli AR. Transgender youth and life-threatening behaviors. Suicide Life Threat Behav 2007; 37: 527-537

[18] Hugh-Jones S, Smith PK. Self-reports of short-and long-term effects of bullying on children who stammer. Br J Educ Psychol 1999; 69: 141-158

[19] Kiess A, Körner A, Kiess W, Seikowsky K et al. Transsexualität im Kindes-und Jugendalter. Kinder- Jugendmed 2012; 12: 212-217

[20] Korte A, Goecker D, Krude $\mathrm{H}$ et al. Gender identity disorders in childhood and adolescence: Currently debated concepts and treatment strategies. Dtsch Ärztebl Int 2008; 105: 834

[21] Landén M, Wälindel J, Lundström B. Incidence and sex ratio of transsexualism in Sweden. Acta Psychiatr Scand 1996; 93: 261-263

[22] Salmon G, James A, Smith DM. Bullying in schools: Self reported anxiety, depression, and self esteem in secondary school children. BM 1998; 317: 924-925
[23] Steensma TD, Cohen-Kettenis PT, Zucker K]. Evidence for a Change in the Sex Ratio of Children Referred for Gender Dysphoria: Data from the Center of Expertise on Gender Dysphoria in Amsterdam (19882016). J Sex Marital Ther 2018; 44: 7, 713-715

[24] Steensma TD, McGuire JK, Kreukels BP et al. Factors associated with desistence and persistence of childhood gender dysphoria: a quantitative follow-up study. J Am Acad Child Adolesc Psychiatry 2013 52: $582-590$

[25] Van Kesteren P], Gooren LJ, Megens JA. An epidemiological and demographic study of transsexuals in the Netherlands. Arch Sex Behav 1996; 25: 589-600

[26] Wallien MS, Swaab H, Cohen-Kettenis PT. Psychiatric comorbidity among children with gender identity disorder. J Am Acad Child Adoles Psychiatry 2007; 46: 1307-1314

[27] Wallien MS, Veenstra R, Kreukels BP et al. Peer group status of gender dysphoric children: A sociometric study. Arch Sex Behav 2010;39: 553-560

[28] Wiepjes CM, Nota NM, de Blok Clet al. The Amsterdam cohort of gender dysphoria study (1972-2015): trends in prevalence, treatment, and regrets. J Sex Med 2018; 15: 582-590

[29] Wood H, Sasaki S, Bradley S] et al. Patterns of referral to a gender identity service for children and adolescents (1976-2011): Age, sex ratio, and sexual orientation. J Sex Marital Ther 2013; 39: 1-6

[30] Zucker KJ, Wilson-Smith DN, Kurita JA et al. Children's appraisals of sex-typed behavior in their peers. Sex Roles 1995: 33: 703-725 


\section{Zusammenfassung}

Dissertation zur Erlangung des akademischen Grades

Dr. med.

Symptome, Komorbiditäten und Therapie von Kindern und Jugendlichen mit Geschlechtsdysphorie

eingereicht von Annika Alica Specht

angefertigt an der Universität Leipzig in der Medizinischen Fakultät

betreut von Prof. Dr. med. Wieland Kiess

Datum der Einreichung 24.11.2020

Geschlechtsdysphorie im Kindes- und Jugendalter zeichnet sich durch ein wachsendes Unbehagen in Bezug auf das biologische Geschlecht aus. Diese Inkongruenz zwischen biologischem und erlebtem Geschlecht verschärft sich häufig mit dem Eintritt in die Pubertät. Geschlechtsdysphorische Kinder sind häufiger als Gleichaltrige von psychiatrischen Komorbiditäten wie Depressionen, selbstverletzendem Verhalten oder Suizidalität betroffen. Die Studie dient der Darstellung der Symptome, der Behandlung sowie der Komorbiditäten von Kindern und Jugendlichen mit Geschlechtsdysphorie, die an der Klinik für Kinder- und Jugendmedizin der Universität Leipzig betreut werden. Der Zeitraum der Studie umfasst die Jahre von 2005 bis 2018. In diesem Zeitraum stellten sich insgesamt 68 Kinder bzw. Jugendliche in der endokrinologischen Sprechstunde aufgrund einer Geschlechtsdysphorie vor. Von diesen schlossen wir 66 in die Studie ein. Die Daten wurden anonymisiert aus den Patientenakten erhoben und anschließend deskriptiv analysiert. Der Behandlungsvertrag am Universitätsklinikum Leipzig beinhaltet eine Einverständniserklärung zum anonymisierten Nutzen von Patientendaten. Für alle erfassten Patient*innen liegt dieser Vertrag und das Einverständnis vor, wogegen die Ethik-Kommission an der medizinischen Fakultät der Universität Leipzig keine ethischen, wissenschaftlichen oder rechtlichen Bedenken erhob. 
Wir stellten einen deutlichen Anstieg der Patientenzahl in den Jahren 2017 und 2018 fest. Während sich in den Jahren von 2005 bis 2011 lediglich ein oder gar kein Kind im Jahr mit Geschlechtsdysphorie vorstellte, erhöhte sich die Zahl 2017 auf 12 und 2018 auf 18 Vorstellungen. Diese Entwicklung deckt sich mit Daten aus einem Behandlungszentrum in Amsterdam, die ebenfalls einen deutlichen Anstieg der Patientenzahlen feststellen konnten.

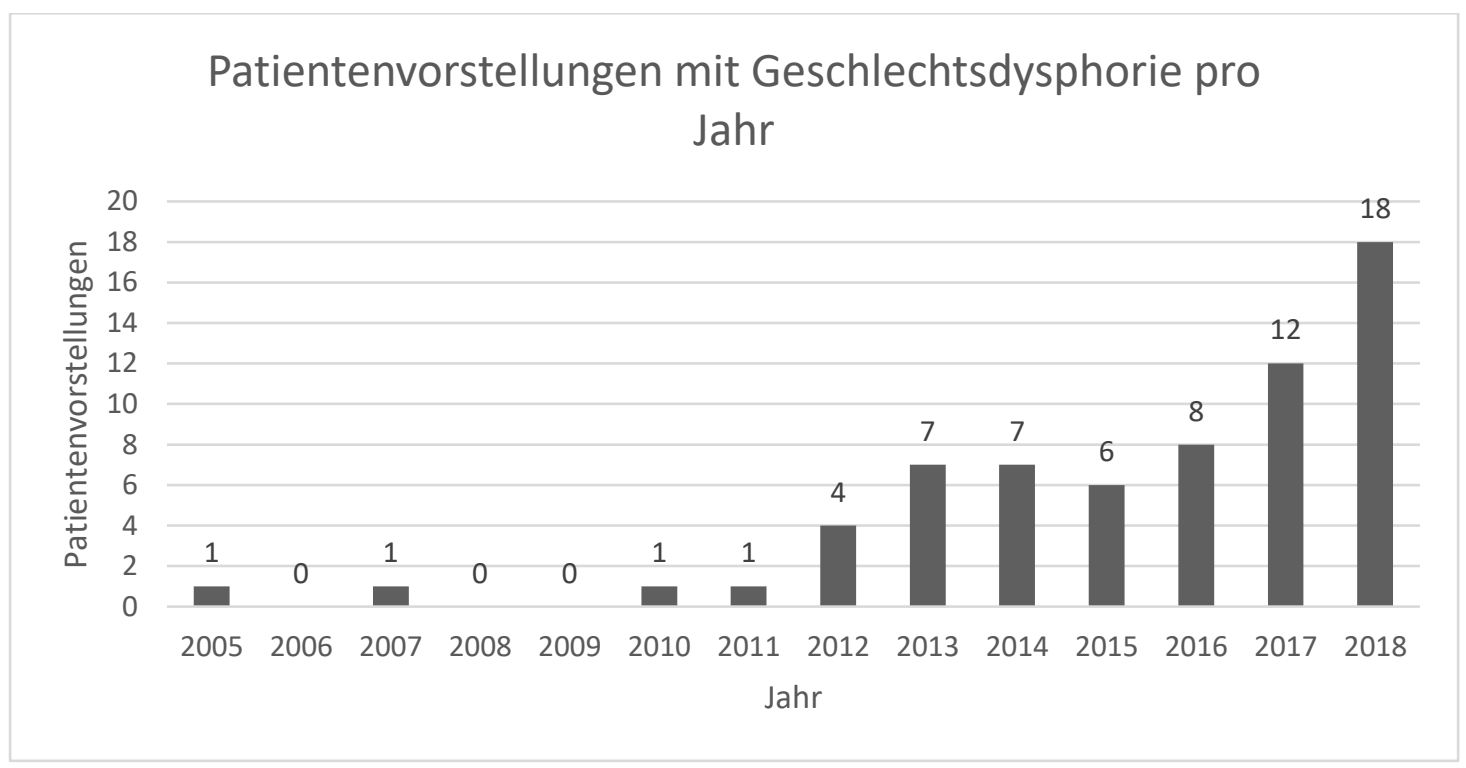

Das Geschlechterverhältnis liegt bei 4,5:1 (w:m). Damit widerspricht unser Ergebnis Studien, die ein Geschlechterverhältnis beschreiben, das teilweise deutlich in Richtung der dem biologischen Geschlecht nach Jungen verschoben ist. Jedoch zeigen aktuellere Studien übereinstimmend die Tendenz der Angleichung des Geschlechterverhältnisses ab der Pubertät. Eine mögliche Ursache könnte eine höhere gesellschaftliche Akzeptanz bzw. Toleranz von jungenhaften Eigenschaften und Verhaltensweisen bei Mädchen als mädchenhaften Eigenschaften und Verhaltensweisen bei Jungen im Kindesalter sein [5, 32, 35]. Dies könnte bedingen, dass Mädchen sich erst mit Eintritt in die Pubertät und der damit aggravierenden psychischen Belastung in Behandlungszentren vorstellen. Das Geschlechterverhältnis gleicht sich auch während der letzten Jahre immer mehr an. Während in der Zeit vor 2000 das Geschlechterverhältnis bezogen von Jungen zu Mädchen mit GD in Toronto bei 5,75:1 und in Amsterdam bei 2,93:1 [8] lag, näherte es sich nach 2000 in Toronto auf 3,41:1 [33] und in Amsterdam auf 1,68:1 (2008 - 2011) [28] an. Diese Theorie wird durch eine deutsche Studie von Garrels et al. [15] und anderen europäischen Studien [12, 23, 30] bestärkt. Neuste Untersuchungen sprechen 
gar von einer Umkehr des Geschlechterverhältnisses [7, 29]. Auch in dieser Arbeit lässt sich eine Veränderung des Geschlechterverhältnisses bezogen auf die Zeit beobachten. So liegt das Verhältnis von Mädchen zu Jungen von Geburtsgeschlecht ausgehend im Zeitraum von 2005 bis 2016 bei 7,2:1. Im Zeitraum von 2017 bis 2018 nähert es sich auf 3,3:1 an. Somit entspricht unser Ergebnis den Tendenzen neuerer Studien [28, 33]. Durchschnittlich lag das Alter der Jugendlichen bei ihrer ersten Vorstellung in der endokrinologischen Sprechstunde bei 13,6 Jahren. Dieses Alter und die damit häufig schon weiter fortgeschrittene körperlicher Entwicklung kann sowohl Ärzt*innen als auch Patient*innen vor Probleme stellen. Die WPATH-SOC [10] empfehlen eine frühe Therapie mit GnRH-Analoga (ab Tanner-Stadium 2), um bessere Behandlungsergebnisse und eine Minderung der psychischen Belastung zu erreichen. Auch Studien zeigten, dass das psychische und operative Ergebnis besser ist, wenn die Pubertät bei Behandlungsbeginn noch nicht zu weit vorangeschritten ist $[9,11]$.

Die Symptome der Geschlechtsdysphorie konnten wir in vier Gruppen gliedern. Diese sind 1. das Verhalten im Spiel, die Wahl des Spielzeugs und die Wahl der Spielpartner*innen/Freund*innen, 2. Die Ablehnung geschlechtsspezifischer Kleidung, 3. die Ablehnung des Geschlechts, Ablehnung des eigenen Körper und den geschlechtsspezifischen körperlichen Veränderungen und 4. die deutliche Äußerung eines Kindes oder Jugendlichen, das Geschlecht wechseln zu wollen.

Anzeichen der Geschlechtsdysphorie (Mehrfachnennungen möglich):

\begin{tabular}{|l|l|}
\hline Anzeichen der Geschlechtsdysphorie & $\mathrm{n}=66(\%)$ \\
\hline $\begin{array}{l}\text { Verhaltensweisen, Freunde und stereotypes Spielzeug des } \\
\text { anderen Geschlechts (\%) }\end{array}$ & $52(78,8)$ \\
\hline Ablehnung geschlechtsspezifischer Kleidung oder Rollen (\%) & $33(50,0)$ \\
\hline Ablehnung des eigenen Geschlechts/der Geschlechtsorgane (\%) & $19(28,8)$ \\
\hline Wunsch/Überzeugung, das Geschlecht wird sich ändern (\%) & $2(3,0)$ \\
\hline
\end{tabular}

Außerdem konnten wir das zeitliche Auftreten der Symptome erheben. Dabei zeigte sich, das bei knapp dreiviertel $(72,7 \%)$ der Kinder die Symptome bereits vor der Pubertät entwickelten, die meisten von ihnen $(93,8 \%)$ bereits vor der Einschulung. 16 
Kinder $(24,2 \%)$ entwickelten erste Symptome mit Eintritt in die Pubertät bzw. im Verlauf der Pubertät.

Die Therapie von Patient*innen mit einer Geschlechtsdysphorie gliedert sich in 3 Phasen: Die reversible Therapie mit GnRH-Analoga, die die Hypophysen-GonadenAchse hemmen und damit das Voranschreiten der Pubertät verhindern, die teilweise reversible Therapie mit Testosteron bzw. Östrogenen, sowie die irreversible chirurgische Therapie, die verschiedene Möglichkeiten der Geschlechtsangleichung bietet. Von den in der Studie betrachteten Patient*innen werden 42 (63,6\%) mit GnRHAnaloga behandelt, 24 dieser Patient*innen $(36,4 \%)$ nehmen geschlechtsangleichende Hormone ein. Operationen zur Geschlechtsangleichung wurden bei fünf der 66 Patient*innen (7,6\%) durchgeführt, was entsprechend dem WPATH-SOC nach stabilem Rollenwechsel und einjähriger Testosterontherapie im Einzelfall möglich ist [10]. Die Therapie mit GnRH-Analoga und geschlechtsangleichenden Hormonen wurde nur von einem Patienten unterbrochen. Die übrigen 41 Patient*innen führten die Therapie nach Beginn weiter, was eine Zufriedenheit mit der Unterdrückung der Pubertät vermuten lässt. Vor oder während der endokrinologischen Behandlung waren 58 der Jugendlichen $(87,9 \%)$ in regelmäßiger psychologischer Betreuung. Wir fanden aufgrund der psychologischen differentialdiagnostischen Untersuchung und der Anamnesen heraus, dass 30 Jugendliche (45,5\%) Anzeichen mindestens einer psychischen Komorbidität auswiesen. Die Häufigkeit und Art der psychischen Komorbiditäten decken sich mit den Ergebnissen anderer Studien $[14,16]$.

Psychiatrische Komorbiditäten (Mehrfachnennungen möglich)

\begin{tabular}{|l|l|}
\hline Psychiatrische Komorbiditäten & $\mathrm{n}=66(\%)$ \\
\hline Essstörung (\%) & $5(7,5)$ \\
\hline Negative Selbstbild (\%) & $14(21,2)$ \\
\hline Selbstverletzendes Verhalten (\%) & $16(24,2)$ \\
\hline Angststörung (\%) & $7(10,6)$ \\
\hline
\end{tabular}




\begin{tabular}{|l|l|}
\hline Depressive Episoden (\%) & $14(21,2)$ \\
\hline Suizidgedanken (\%) & $9(13,9)$ \\
\hline Suizidversuch (\%) & $1(1,5)$ \\
\hline
\end{tabular}

Kinder und Jugendliche, bei denen die Pubertätsentwicklung bei Erstvorstellung bereits über das Stadium P3/G3/B3 nach Tanner überschritten war, leiden häufiger unter Komorbiditäten als Kinder und Jugendliche, die sich früher vorgestellt haben und dementsprechend früher therapiert werden konnten. $\mathrm{Zu}$ beachten ist allerdings, dass nur schwer zu trennen ist, welchen Ursprung die psychischen Komorbiditäten haben. Daher ist davon auszugehen, dass einige Komorbiditäten ebenso aus eigener Unsicherheit und sekundären Interaktionsproblemen mit dem familiären und sozialen Umfeld resultieren können.

Auch die familiären Verhältnisse und die Reaktionen der Eltern auf ein Coming-Out ihrer Kinder legen nahe, dass eine Familientherapie wichtige Unterstützung für die Patient*innen sein kann. So haben insgesamt 16 Jugendliche $(24,2 \%)$ negative Reaktionen in Bezug auf ihre Geschlechtsdysphorie innerhalb der eigenen Familie erfahren und berichteten von Ablehnung eines oder mehrerer Familienmitglieder. Erfahrungen mit körperlicher Misshandlung wurde von drei Kindern (4,5\%) berichtet, wobei aus der Anamnese nicht hervorgeht, in welchem Zusammenhang diese zur Geschlechtsdysphorie steht. Auch andere Studien haben gezeigt, dass Kinder und Jugendliche mit GD häufiger Opfer verbaler und körperlicher Gewalt werden und diese sich negativ auf die psychische Gesundheit auswirkt $[1,16]$.

Im Freundeskreis haben die Kinder und Jugendlichen häufig positive Reaktionen nach ihrem Outing erhalten, jedoch berichten 17 Jugendliche $(25,8 \%)$ von Mobbing aufgrund ihrer Geschlechtsdysphorie. Studien zeigen, dass Mobbing ein niedriges Selbstbewusstsein, Einsamkeit, Depressionen, Suizidgedanken und -versuche und Suizide hervorrufen kann [26]. Diese Erfahrungen können die weitere Schulzeit bis hin ins Erwachsenenalter beeinflussen [19] und sollten daher im ärztlichen Gespräch thematisiert werden.

Durch diese Studie soll dargestellt werden, wie komplex und interdisziplinär die Betreuung von Kindern und Jugendlichen mit Geschlechtsdysphorie ist. Gleichzeitig 
sollte hervorgehoben werden, wie einschneidend und prägend eine Geschlechtsdysphorie für die Betroffenen und ihre Familien ist, besonders wenn spezialisierte Hilfe fehlt. Daher ist es wünschenswert, Strukturen zu schaffen, die es Patient*innen und Eltern, aber auch niedergelassenen Pädiater*innen erleichtern, Hilfe von spezialisierten Zentren zu erhalten. Das bietet die Möglichkeit der frühzeitigen Anbindung, Aufklärung über Behandlungsoptionen, Unterstützung bei akuten Belastungssituationen, die z.B. im Rahmen des Outings entstehen können sowie die Prävention als auch frühzeitige Therapie möglicher Komorbiditäten. 


\section{$4 \quad$ Literaturverzeichnis}

[1] Almeida, Joanna, et al. "Emotional distress among LGBT youth: The influence of perceived discrimination based on sexual orientation." Journal of youth and adolescence 38.7 (2009): 1001-1014.

[2] American Psychiatric Association. Diagnostic and statistical manual of mental disorders (DSM-5®). American Psychiatric Pub, 2013.

[3] Arbeitsgemeinschaft der Wissenschaftlichen Medizinischen Fachgemeinschaften (AWMF)-Ständige Kommission der Leitlinien. AWMFRegister “Leitlinien” Registernummer 138 - 001, Stand: 09.10.2018, gültig bis 08.10.2023, verfügbar: https://www.awmf.org/leitlinien/detail/11/138-001.html, Zugriff am 10.02.2020

[4] Arbeitsgemeinschaft der Wissenschaftlichen Medizinischen Fachgemeinschaften (AWMF)-Ständige Kommission der Leitlinien. AWMFRegister “Leitlinien” Registernummer 028 - 014, Anmeldedatum: 28.09.2015, geplante Fertigstellung: 27.09.2020, verfügbar: https://www.awmf.org/leitlinien/detail/anmeldung/1/11/028-014.html

[5] Blakemore, Judith E. Owen. "Children's beliefs about violating gender norms: Boys shouldn't look like girls, and girls shouldn't act like boys." Sex roles 48.910 (2003): 411-419.

[6] Bosinski, Hartmut AG, et al. "Geschlechtsidentitätsstörungen bei Kindern und Jugendlichen Nosologie und Epidemiologie." Monatsschrift Kinderheilkunde 144.11 (1996): 1235-1241.

[7] Carmichael, P., T. D. Steensma, and K. J. Zucker. "Evidence for a Change in the Sex Ratio of Children Referred for Gender Dysphoria: Data From the Gender Identity Development Service in London (2000-2017)." The journal of sexual medicine 15.10 (2018): 1381-1383.

[8] Cohen-Kettenis, Peggy T., et al. "Demographic characteristics, social competence, and behavior problems in children with gender identity disorder: A 
cross-national, cross-clinic comparative analysis." Journal of abnormal child psychology 31.1 (2003): 41-53.

[9] Cohen-Kettenis, Peggy T., et al. "Puberty suppression in a gender-dysphoric adolescent: a 22-year follow-up." Archives of sexual behavior 40.4 (2011): 843847.

[10] Coleman, Eli, et al. "Standards of care for the health of transsexual, transgender, and gender-nonconforming people, version 7." International journal of transgenderism 13.4 (2012): 165-232.

[11] Delemarre-Van De Waal, Henriette A., and Peggy T. Cohen-Kettenis. "Clinical management of gender identity disorder in adolescents: a protocol on psychological and paediatric endocrinology aspects." European Journal of Endocrinology 155.suppl_1 (2006): S131-S137.

[12] Dixen, Jean M., et al. "Psychosocial characteristics of applicants evaluated for surgical gender reassignment." Archives of Sexual Behavior 13.3 (1984): 269276.

[13] Drummond, Kelley D., et al. "A follow-up study of girls with gender identity disorder." Developmental psychology 44.1 (2008): 34.

[14] Fitzpatrick, Kathleen Kara, et al. "Gender role, sexual orientation and suicide risk." Journal of affective disorders 87.1 (2005): 35-42.

[15] Garrels, Lutz, et al. "Sex ratio of transsexuals in Germany: the development over three decades." Acta Psychiatrica Scandinavica 102.6 (2000): 445-448.

[16] Grossman, Arnold H., and Anthony R. D'Augelli. "Transgender youth and lifethreatening behaviors." Suicide and Life-Threatening Behavior 37.5 (2007): $527-537$.

[17] Grossman, Arnold H., et al. "Comparing gender expression, gender nonconformity, and parents' responses of female-to-male and male-to-female transgender youth: Implications for counseling." Journal of LGBT Issues in Counseling 1.1 (2006): 41-59. 
[18] Hembree, Wylie C., et al. "Endocrine treatment of transsexual persons: an Endocrine Society clinical practice guideline." The Journal of Clinical Endocrinology \& Metabolism 94.9 (2009): 3132-3154.

[19] Hugh-Jones, Siobhan, and Peter K. Smith. "Self-reports of short-and long-term effects of bullying on children who stammer." British Journal of Educational Psychology 69.2 (1999): 141-158.

[20] Korte, Alexander, et al. "Gender identity disorders in childhood and adolescence: currently debated concepts and treatment strategies." Deutsches Ärzteblatt International 105.48 (2008): 834.

[21] Kuiper, Bram, and Peggy Cohen-Kettenis. "Sex reassignment surgery: a study of 141 Dutch transsexuals." Archives of sexual behavior 17.5 (1988): 439-457.

[22] Kuyper, Lisette, and Ciel Wijsen. "Gender identities and gender dysphoria in the Netherlands." Archives of sexual behavior 43.2 (2014): 377-385.

[23] Landén, M., J. Wålindel, and B. Lundström. "Incidence and sex ratio of transsexualism in Sweden." Acta Psychiatrica Scandinavica 93.4 (1996): 261 263.

[23] Meyenburg, Bernd, Anne Kröger, and Rebecca Neugebauer. "Transidentität im Kindes- und Jugendalter." Zeitschrift für Kinder- und Jugendpsychiatrie und Psychotherapie (2014).

[24] Russell, Stephen T. "Sexual minority youth and suicide risk." American Behavioral Scientist 46.9 (2003): 1241-1257.

[25] Salmon, Gilly, Anthony James, and David M. Smith. "Bullying in schools: self reported anxiety, depression, and self esteem in secondary school children." $\mathrm{BMj}$ 317.7163 (1998): 924-925.

[26] Steensma, Thomas D., et al. "Desisting and persisting gender dysphoria after childhood: a qualitative follow-up study." Clinical child psychology and psychiatry 16.4 (2011): 499-516. 
[27] Steensma, Thomas D., et al. "Factors associated with desistence and persistence of childhood gender dysphoria: a quantitative follow-up study." Journal of the American Academy of Child \& Adolescent Psychiatry 52.6 (2013): 582-590.

[28] Steensma, Thomas D., Peggy T. Cohen-Kettenis, and Kenneth J. Zucker. "Evidence for a change in the sex ratio of children referred for gender dysphoria: data from the Center of Expertise on Gender Dysphoria in Amsterdam (19882016)." Journal of sex \& marital therapy 44.7 (2018): 713-715.

[29] Van Kesteren, Paul J., Louis J. Gooren, and Jos A. Megens. "An epidemiological and demographic study of transsexuals in the Netherlands." Archives of sexual behavior 25.6 (1996): 589-600.

[30] Wallien, Madeleine SC, and Peggy T. Cohen-Kettenis. "Psychosexual outcome of gender-dysphoric children." Journal of the American Academy of Child \& Adolescent Psychiatry 47.12 (2008): 1413-1423.

[31] Wallien, Madeleine SC, et al. "Peer group status of gender dysphoric children: A sociometric study." Archives of Sexual Behavior 39.2 (2010): 553-560.

[32] Wood, Hayley, et al. "Patterns of referral to a gender identity service for children and adolescents (1976-2011): age, sex ratio, and sexual orientation." Journal of sex \& marital therapy 39.1 (2013): 1-6.

[33] World Health Organization. "The ICD-10 classification of mental and behavioural disorders: clinical descriptions and diagnostic guidelines." Weekly Epidemiological Record= Relevé épidémiologique hebdomadaire 67.30 (1992): 227-227.

[34] Zucker, Kenneth J., et al. "Children's appraisals of sex-typed behavior in their peers." Sex Roles 33.11-12 (1995): 703-725. 


\section{Darstellung des eigenen Beitrags, Anteil der Koautoren}

Annika Alica Specht (Erstautorin):

- Entwicklung der Studienidee

- Datenerhebung

- Datenauswertung und -interpretation

- Verfassen und Überarbeiten des Manuskripts

Julia Gesing:

- Entwicklung der Studienidee

- Unterstützung bei der Datenerhebung

- Verfassen und Überarbeiten des Manuskripts

Roland Pfäffle:

- Überarbeiten des Manuskripts

Antje Körner:

- Überarbeiten des Manuskripts

Wieland Kiess:

- Entwicklung der Studienidee

- Datenauswertung und -interpretation

- Überarbeiten des Manuskripts

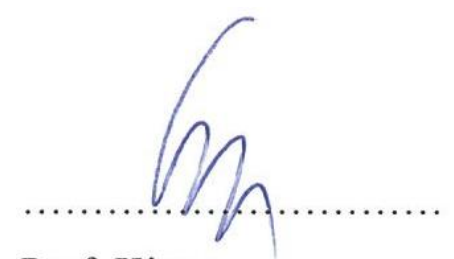

Prof. Kiess

Dr. med. Gesing

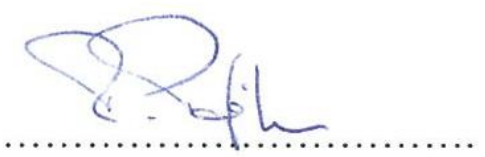

Prof. Pfäffle

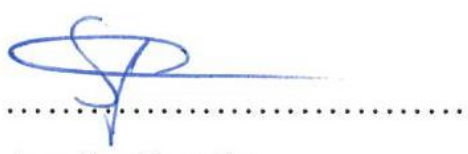

Annika Specht 
Prof. Körner 


\section{Erklärung über die eigenständige Abfassung der Arbeit}

Hiermit erkläre ich, dass ich die vorliegende Arbeit selbstständig und ohne unzulässige Hilfe oder Benutzung anderer als der angegebenen Hilfsmittel angefertigt habe. Ich versichere, dass Dritte von mir weder unmittelbar noch mittelbar eine Vergütung oder geldwerte Leistungen für Arbeiten erhalten haben, die im Zusammenhang mit dem Inhalt der vorgelegten Dissertation stehen, und dass die vorgelegte Arbeit weder im Inland noch im Ausland in gleicher oder ähnlicher Form einer anderen Prüfungsbehörde zum Zweck einer Promotion oder eines anderen Prüfungsverfahrens vorgelegt wurde. Alles aus anderen Quellen und von anderen Personen übernommene Material, das in der Arbeit verwendet wurde oder auf das direkt Bezug genommen wird, wurde als solches kenntlich gemacht. Insbesondere wurden alle Personen genannt, die direkt an der Entstehung der vorliegenden Arbeit beteiligt waren. Die aktuellen gesetzlichen Vorgaben in Bezug auf die Zulassung der klinischen Studien, die Bestimmungen des Tierschutzgesetzes, die Bestimmungen des Gentechnikgesetzes und die allgemeinen Datenschutzbestimmungen wurden eingehalten. Ich versichere, dass ich die Regelungen der Satzung der Universität Leipzig zur Sicherung guter wissenschaftlicher Praxis kenne und eingehalten habe.

Datum

Unterschrift 


\section{$7 \quad$ Lebenslauf}

Name

Geburts-

datum /-ort

Anschrift

Telefon

E-Mail
Annika Alica Specht

09.10.1992 in Mönchengladbach

Brückenstraße 3, 86153 Augsburg

+4915224580568

annikaalica.specht@gmail.com

Berufs- $\quad$ Seit 05.2020 Weiterbildung zur Viszeralchirurgin am

tätigkeit

Universitätsklinikum Augsburg

Studium 10.2012 bis

Studium der Humanmedizin an der Universität Leipzig

12.2019

12.2019

Abschluss des Medizinstudiums an der Universität Leipzig, Gesamtnote 2,0

08.2014 Erster Abschnitt der ärztliche Prüfung, Note 3

10.2018 Zweiter Abschnitt der ärztlichen Prüfung, Note 2

12.2019 Dritter Abschnitt der ärztlichen Prüfung, Note 1

Promotion 2015 bis 2020

Promotion an der Klinik und Poliklinik

für Kinder - und Jugendmedizin,

Universitätsklinikum Leipzig

Thema: Symptome, Komorbiditäten und

Therapie von Kindern und Jugendlichen

mit Geschlechtsdysphorie

11.2019

Manuskript eingereicht und angenommen

in der Fachzeitschrift „Klinische

Pädiatrie“ des Georg Thieme Verlags 
Datum

Unterschrift 


\section{$8 \quad$ Danksagung}

An dieser Stelle möchte ich mich herzlich bei allen Menschen bedanken, die diese Arbeit ermöglicht haben.

In erster Linie möchte ich mich bei Herrn Prof. Kiess bedanken, der mir die Publikation in der Klinik und Poliklinik für Kinder und Jugendliche der Universitätsklinik Leipzig überhaupt erst ermöglicht hat und mir jederzeit mit Rat und Unterstützung zu Seite stand.

Weiterer Dank gebührt Frau Dr. Gesing, die mich in der Erarbeitung des Manuskripts geduldig unterstützt hat und ihre Expertise in der Betreuung der Patienten mit mir geteilt hat.

Ich möchte Herrn Prof. Pfäffle danken, der mir bei der Erhebung der Daten geholfen hat.

Und nicht zuletzt danke ich meiner Familie und meinen Freunden für die emotionale Unterstützung, die maßgeblich dazu beigetragen hat, diese Arbeit fertigzustellen.

Vielen Dank 
